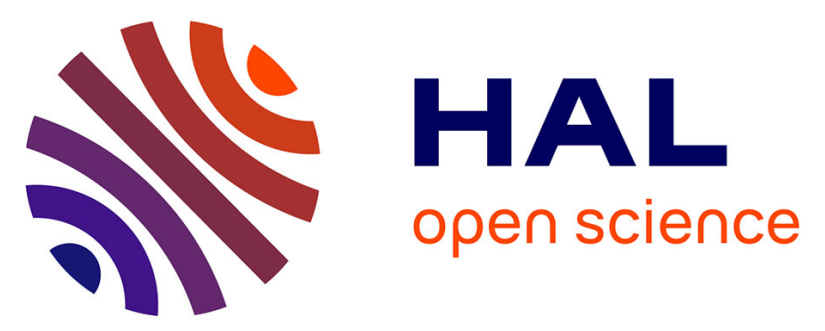

\title{
Retombées volcaniques dans des tourbières et lacs autour du massif des Nevados Ampato et Sabancaya (Pérou méridional, Andes Centrales)
}

Etienne Juvigné, Jean-Claude Thouret, Isabelle Loutsch, Sébastien Lamadon, Manfred Frechen, Michel Fontugne, Marco Rivera, Jasmine Dávila, Jersy Mariño

\section{To cite this version:}

Etienne Juvigné, Jean-Claude Thouret, Isabelle Loutsch, Sébastien Lamadon, Manfred Frechen, et al.. Retombées volcaniques dans des tourbières et lacs autour du massif des Nevados Ampato et Sabancaya (Pérou méridional, Andes Centrales). Quaternaire, 2008, Téphras et séquences quaternaires, 19 (2), pp.157-173. 10.4000/quaternaire.3362 . hal-02470528

\section{HAL Id: hal-02470528 \\ https://hal.science/hal-02470528}

Submitted on 19 Oct 2020

HAL is a multi-disciplinary open access archive for the deposit and dissemination of scientific research documents, whether they are published or not. The documents may come from teaching and research institutions in France or abroad, or from public or private research centers.
L'archive ouverte pluridisciplinaire HAL, est destinée au dépôt et à la diffusion de documents scientifiques de niveau recherche, publiés ou non, émanant des établissements d'enseignement et de recherche français ou étrangers, des laboratoires publics ou privés. 


\title{
RETOMBÉES VOLCANIQUES DANS DES TOURBIÈRES ET LACS AUTOUR DU MASSIF DES NEVADOS AMPATO ET SABANCAYA (PÉROU MÉRIDIONAL, ANDES CENTRALES)
}

\author{
Etienne JUVIGNÉ ${ }^{1}$, Jean-Claude THOURET ${ }^{2}$, Isabelle LOUTSCH ${ }^{1}$, \\ Sébastien LAMADON ${ }^{2}$, Manfred FRECHEN ${ }^{3}$, Michel FONTUGNE ${ }^{4}$, \\ Marco RIVERA ${ }^{5}$, Jasmine DÁVILA ${ }^{6} \&$ Jersy MARIÑO ${ }^{5}$
}

\begin{abstract}
RÉSUMÉ
Des recherches multidisciplinaires ont été réalisées à partir de coupes et carottes extraites de tourbières et d'un lac, au Pérou méridional, dans la région volcanique du massif des Nevados Ampato, Sabancaya et Hualca Hualca, proche du canyon du Río Colca et de la ville d’Arequipa. L'objectif majeur de la présente recherche est de compléter la partie holocène d'un modèle téphrostratigraphique du Pérou méridional, portant sur les cinquante derniers milliers d'années. Les sites étudiés sont plus particulièrement la grande tourbière de Sallalli et les rives de la Laguna Mucurca. La tourbière de Sallalli se trouve sur le piémont oriental du Massif Ampato-Sabancaya ; elle a été barrée par des moraines remontant à la dernière glaciation ainsi que par des coulées de lave au SE. La séquence tourbeuse de Sallalli comprend au moins cinq téphras. Les niveaux les plus récents consistent en retombées pliniennes de verres rhyolithiques dont l'attribution est discutée entre l'éruption du Huaynaputina en 1600AD et une autre du Misti qui a eu lieu entre $800 \mathrm{cal} \mathrm{BC}$ et $200 \mathrm{cal} \mathrm{AD}$. Les autres téphras sont des produits hétérogènes d'origine phréatomagmatique émis probablement par le Sabancaya, voire l'Ampato pendant les périodes respectives suivantes : $150 \mathrm{cal} \mathrm{AD}$ à $100 \mathrm{cal} \mathrm{BC}, 150$ à $420 \mathrm{cal} \mathrm{BC}, 2100$ à $2500 \mathrm{cal} \mathrm{BC}$ et 7420 à $7750 \mathrm{cal} \mathrm{BC}$. Les dépôts lacustres récents de la Laguna Mucurca à l'ouest du Nevado Ampato renferment notamment un téphra hétérogène d'une éruption phréatomagmatique, qui aurait eu lieu entre 920 et 760 cal BC; il serait issu de l'un des cônes proches, situé dans le champ des volcans monogénétiques de Huambo sur la marge sud du Río Colca.
\end{abstract}

Mots-clés : Andes centrales, Pérou, Nevado Ampato, Nevado Sabancaya, volcans, téphrostratigraphie, téphrochronologie, ${ }^{14} \mathrm{C}$, Holocène.

\section{ABSTRACT}

VOLCANIC ASH-FALLS IN PEAT-BOG AND LAKES AROUND NEVADOS AMPATO AND SABANCAYA MASSIF (SOUTHERN PERU, CENTRAL ANDES)

Multidisciplinary investigations have been carried out on sections and sediment cores from a lake and peat bogs in the Western Cordillera in the Central Andes of Southern Peru, around Nevados Ampato, Sabancaya and Hualca Hualca volcanic massif. More than 850,000 inhabitants of the city of Arequipa and the Colca valley are concerned by further activities of those volcanoes. The aim of the current study is to complete the Holocene tephrostratigraphical model for southern Peru in which about 40 tephras are distributed within the past 50,000 years. The tephras described in this paper have been discovered in two sites: the large peat-bog of Sallalli at the southern piedmont of the Sabancaya volcano, and the Laguna Mucurca, a salar (brine lake) $15 \mathrm{~km} \mathrm{~W}$ of Nevado Ampato. The Sallalli peat-bog is located in a glacially shaped piedmont, which has been dammed by recent moraines and lava flows $10 \mathrm{~km} \mathrm{SE}$ of the summit of Nevado Sabancaya. The peat section, which spans a large part of the Holocene period (since ca. $9650 \mathrm{yr}$ ), encompasses at least five tephras. The most recent ones consist in plinian ash-falls caracterized by rhyolitic glass shards that can be linked either with the AD 1600 eruption of the Huaynaputina volcano (120 km to the ESE) or with an eruption of the Misti volcano $(60 \mathrm{~km}$ to the SE) that occurred between $800 \mathrm{cal} \mathrm{BC}$ and $200 \mathrm{cal} \mathrm{AD}$. The other tephras consist in phreotomagmatic products of local origin likely erupted by the Sabancaya volcano, or by the Ampato volcano, within the periods bracketed as follows: (i) 150 cal AD and 100 cal BC; (ii) 150 and 420 cal BC; (iii) 2100 and $2500 \mathrm{cal} \mathrm{BC}$; and (iv) 7420 et $7750 \mathrm{cal} \mathrm{BC}$. The recent lacustrine deposits of the Laguna Mucurca host traces of a plinian ash-fall from Huaynaputina volcano and a scoriaceous tephra of phreatomagmatic origin. The latter was emplaced between 920 and 760 cal BC. This tephra has probably been produced by one of the neighbouring cones located in the field of monogenic volcanoes close to Huambo on the southern edge of the Río Colca valley.

Key-words: Central Andes, Peru, Nevado Ampato, Nevado Sabancaya, volcanoes, tephrostratigraphy, chronology, Holocene.

\footnotetext{
${ }^{1}$ Laboratoire de Géologie du Quaternaire, Université de Liège, Sart Tilman, Bât 12A, 4000, Liège, Belgique. Couriel : ejuvigne@ulg.ac.be

${ }^{2}$ Laboratoire Magmas et Volcans, UMR 6524 CNRS, OPGC et IRD, Université Blaise Pascal, 5 rue Kessler, F-63038 Clermont-Ferrand cedex,

France.Courriel : thouret@opgc.univ-bpclermont.fr

${ }_{3}^{3}$ Geochronology and Isotope Hydrology, Universität Hannover, Stilleweg 2, D-30655 Hannover, Deutschland

${ }^{4}$ LSCE, Laboratoire des Sciences du Climat et de l'Environnement, Domaine du CNRS, Bât. 12, 91198 Gif-sur-Yvette cedex, France.

${ }_{5}^{5}$ INGEMMET, Instituto Nacional Geologico-Minero y Metalurgico, Avenida Canada, San Borja, Lima, Peru.

${ }^{6}$ IGP, Instituto Geofísico del Perú, Lima, Peru.
} 


\section{1 - INTRODUCTION}

Des recherches téphrostratigraphiques dans le cadre morpho-sédimentaire glaciaire et périglaciaire de la Cordillère occidentale du Pérou méridional (fig. 1) ont permis de découvrir plus de 40 téphras recouvrant une période de 50.000 ans (tab. 1). Ce programme mené depuis plusieurs années met notamment en exergue les risques encourus par un million d'habitants vivant dans cette zone (Thouret et al., 2001, 2002a).

Une dizaine de volcans actifs ou potentiellement actifs sont situés à une distance de 20 à $150 \mathrm{~km}$ des villes d'Arequipa (850 000 habitants), Moquegua et Tacna (200 000 personnes chacune) et de nombreuses villes plus petites. Neuf volcans sont réputés actifs : Nevado Sara Sara, Nevado Sabancaya, El Misti, Ubinas, Huaynaputina, Ticsani, Nevado Tutupaca, Yucamane et Casiri (fig. 1). Six d'entre eux (Huaynaputina, Misti, Ubinas, Nevado Sabancaya, Nevado
Tutupaca et Yucamane) ont connu au moins une éruption depuis la conquête espagnole en 1540. Par le fait qu'ils sont couverts de glace et de neige, ces volcans « Nevados » représentent nécessairement une menace quotidienne en cas d'éruption, voire même simplement de réchauffement. La vallée du Colca où habitent environ 30000 personnes est devenue une destination touristique importante. Elle est particulièrement exposée à l'activité du Nevado Sabancaya, dont la dernière éruption, vulcanienne, a commencé en 1986 et a duré jusqu'en 1998 (Thouret et al., 1994). Après 1998, son activité a considérablement diminué (Gerbe \& Thouret, 2004). Depuis mars 2006, 1'Ubinas est entré dans une crise éruptive phréatique puis vulcanienne modérée, qui dure encore aujourd'hui (Thouret et al., 2005). L'éruption plinienne et ignimbritique du Huaynaputina en 1600 AD, la plus importante dans toutes les Andes durant l'histoire récente, a produit un volume de téphra de $12 \mathrm{~km}^{3}$ (Thouret et al., 1997, 2002b) à $20 \mathrm{~km}^{3}$ (Adams et al., 2001).

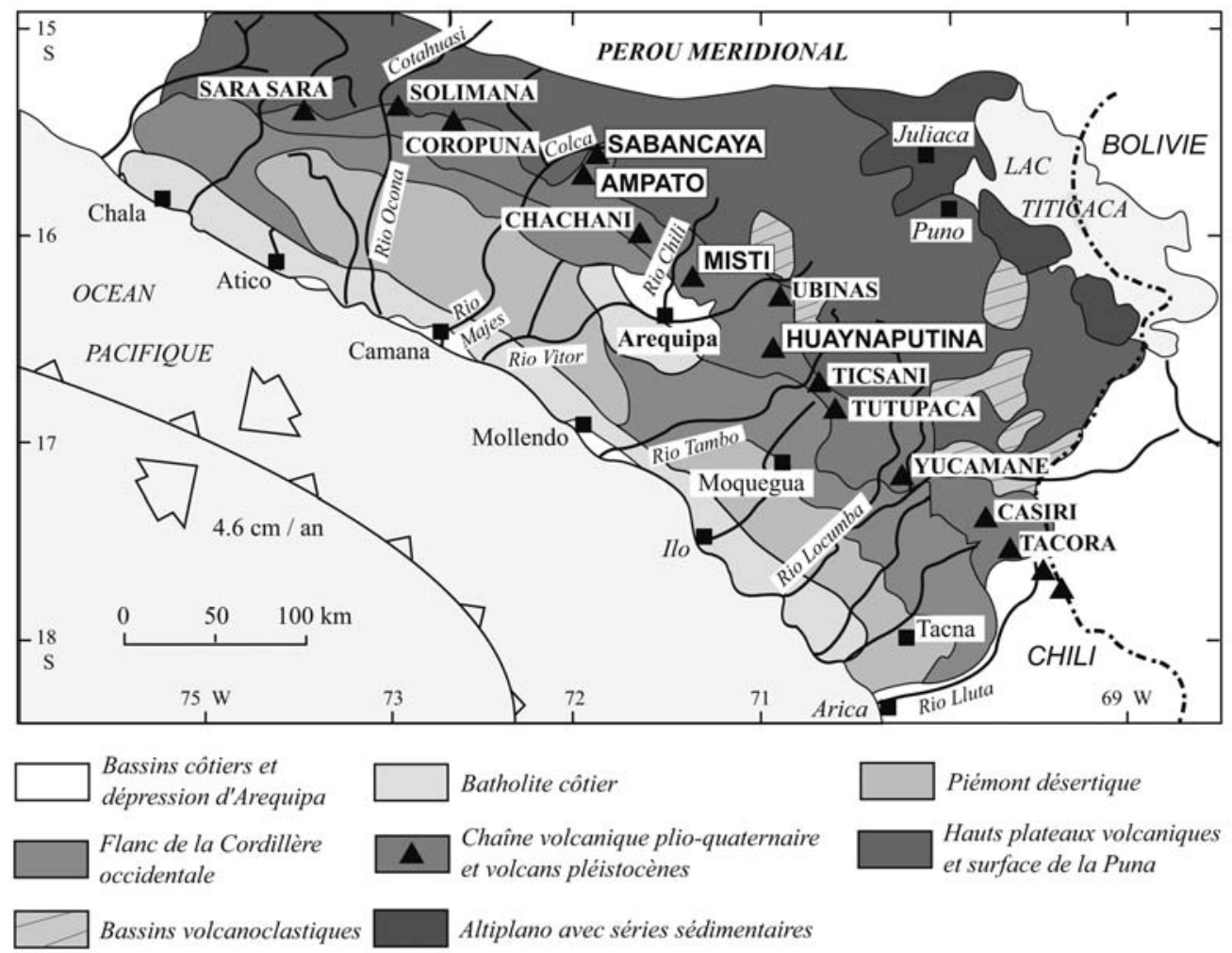

Fig. 1 : Localisation de la chaîne volcanique pléistocène et holocène au Pérou méridional (Thouret et al., 2001).

Fig. 1: Location of the volcanic range of Pleistocene and Holocene age in southern Peru (Thouretet al., 2001).

Tab. 1 : Stratigraphie et chronologie des téphras du Pléistocène supérieur et de l'Holocène découverts dans la chaîne volcanique au sud du Pérou (Thouret et al., 2002a). Les rectangles gris mettent en exergue les huit dépôts de retombées de ponces les plus dispersés $\left(>1000 \mathrm{~km}^{2}\right)$ et les plus volumineux $\left(\geq 1 \mathbf{~ k m}^{3}\right)$. Les téphras du tableau 1 ont été trouvés autour de onze strato-volcans, dômes ou champs de lave dans la Cordillère occidentale, du nord-ouest au sud-est (fig. 1,2 et 3) : (1) le volcan subactif Nevado Sara Sara (5600 m) à 180 km à l'WNW d'Arequipa ; (2) le complexe de dômes dormant du Nevado Coropuna $(6380 \mathrm{~m})$; (3) le champ monogénétique de Orcopampa-Andahua-Huambo à mi-distance des Nevados Coropuna et Ampato-Sabancaya (Delacour et al., 2007) ; (4) le Nevado Sabancaya, dômes actifs (5980 m) et le strato-volcan Nevado Ampato, subactif ou dormant (fig. 4 ; Gerbe \& Thouret, 2004); (5) le volcan actif (fumerollien) El Misti (5820 m) dont le cratère est à $17 \mathrm{~km}$ du centre de la ville d'Arequipa et dont la dernière éruption date du XV' siècle (Thouret et al., 2001) ; (6) le salar (lac salé) de la Laguna Salinas $(4300 \mathrm{~m})$ à mi-distance des édifices El Misti et Ubinas ; (7) l'Ubinas (5600 m), appareil le plus actif du Pérou au cours de l'histoire (Thouret et al., 2005) ; (8) le Huaynaputina $(4600 \mathrm{~m})$, site de la plus grande éruption historique dans les Andes en 1600 (Thouret et al., 1999) ; (9) Les dômes fumerolliens du Ticsani $(5470 \mathrm{~m})$, au nord de la ville de Moquegua ; (10) le volcan actif Nevado Tutupaca (5815 m) à l'est du Tiscsani ; (11) le volcan actif Yucamane (5450 m) près de la ville de Candarave au NE de Moquegua. 


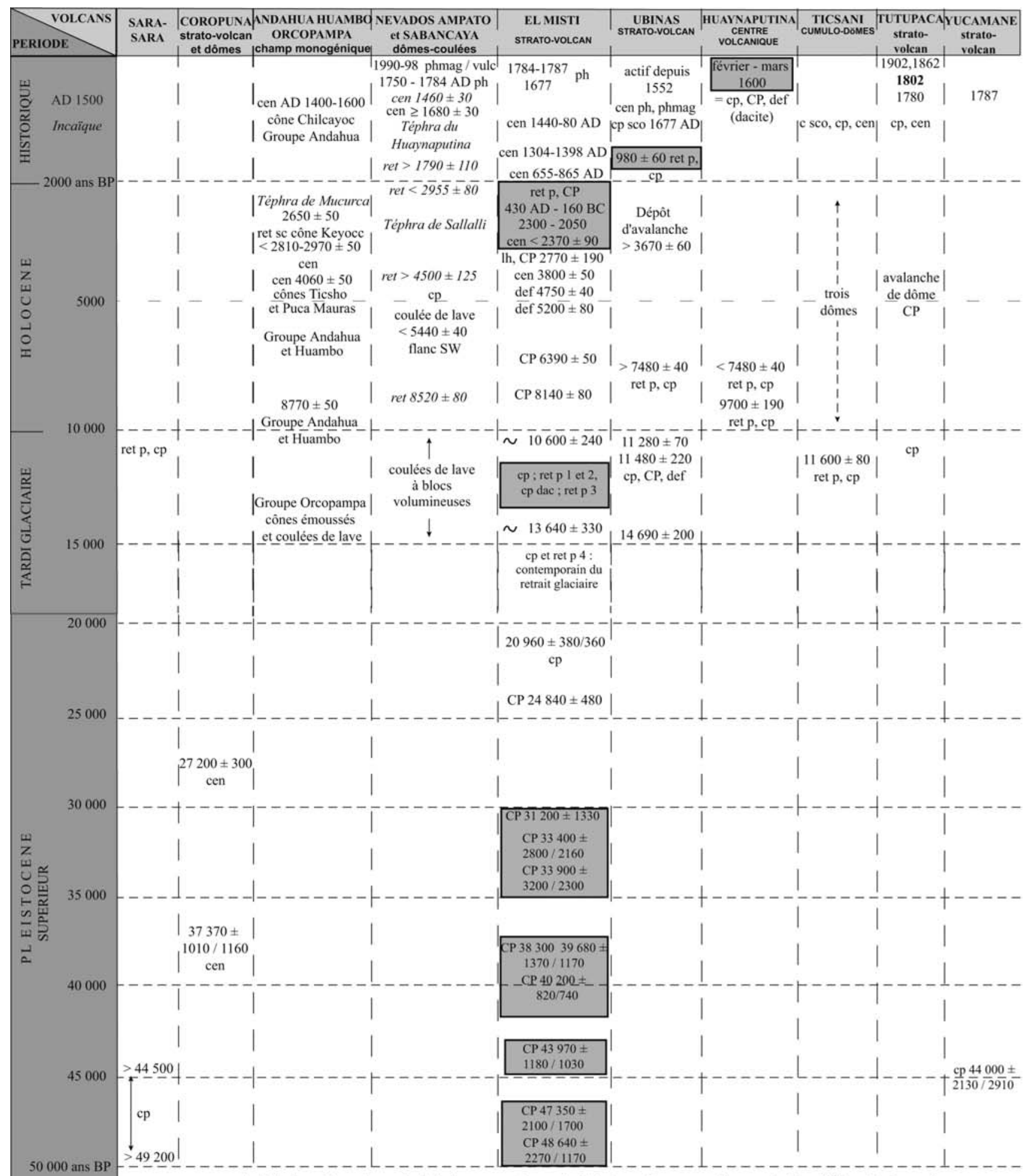

cen : retombée de cendre ; ret $\mathrm{p}$ : retombée de ponce ; ret $\mathrm{sc}$ : retombée de scories ; $\mathrm{cp}$ : coulée pyroclastique $(\mathrm{CP}=$ volumineuse $)$;

def : déferlante pyroclastique ; $\mathrm{c}$ sco coulée de scories et cendres; ph : phreatique ; phmag: phreatomagmatique ; st : strombolien ; and andésite ; dac dacite. $\square$ dépôt volumineux de retombée $>1 \mathrm{~km}^{3}$; en italiques : téphras identifiés dans le massif de l'Ampato-Sabancaya (cet article).

Tab. 1: Stratigraphy and chronology of upper Pleistocene and Holocene tephras, which have been found in the volcanic range in southern Peru (Thouret et al., 2002a). Grey boxes highlight the eight most dispersed $\left(>1000 \mathrm{~km}^{2}\right)$ and most voluminous $\left(\geq 1 \mathrm{~km}^{3}\right)$ pumice-fall deposits. Tephras of table 1 have been identified around eleven stratovolcanoes, domes or lava fields in the Western Cordillera, that are, from Northwest to Southeast (fig. 1, 2 and 3): (1) the subactive Nevado Sara Sara volcano $(5600 \mathrm{~m}) 180 \mathrm{~km}$ to the WNW of Arequipa; (2) the dormant dome complex that forms Nevado Coropuna $(6380 \mathrm{~m}$ ): (3) the monogenetic field of Orcopampa-Andahua-Huambo at mid-distance between Nevados Coropuna and Ampato-Sabancava (Delacour et al., 2007); (4) Nevado Sabancaya that consists of active domes (5980 m), and the subactive or dormant Nevado Ampato stratovolcano (fig. 4; Gerbe \& Thouret, 2004); (5) the active (fumarolic) El Misti stratovolcano $(5820 \mathrm{~m}$ ) whose crater lies $17 \mathrm{~km}$ only away from the city centre of Arequipa, and which last erupted by mid XVth century (Thouret et al., 2001); (6) the salar (brine lake) of Laguna Salinas (4300 m) at mid-distance between El Misti and Ubinas edifices; (7) Ubinas, the most historically active stratovolcano (5600 m) in southern Peru (Thouret et al., 2005); (8) Huaynaputina (4600 $\mathrm{m}$ ), the site of the largest historical eruption in the Andes, which occurred in AD 1600 (Thouret et al., 1999); (9) the fumarolic domes of Ticsani (5470 m), north of the city of Moquegua; (10) the active Nevado Tutupaca stratovolcano $(5815 \mathrm{~m})$ east of Tiscsani; (11) the active Yucamane stratovolcano $(5450 \mathrm{~m})$ near the city of Candarave NE of Moquegua. 
Cet article décrit des téphras holocènes découverts dans une tourbière et un lac salé sur les piémonts est et nord-ouest du massif des Nevados Ampato et Sabancaya. Il enrichit en cela les résultats acquis antérieurement dans le projet TESSOPE (Thouret et al., 2000, 2002a).

\section{2 - LE MASSIF DE L'AMPATO-SABANCAYA- HUALCA HUALCA}

Le massif de l'Ampato-Sabancaya-Hualca Hualca s'est édifié à proximité du versant méridional du canyon du Rio Colca (fig. 2). Le Nevado Ampato est un grand strato-volcan que le Nevado Sabancaya prolonge vers le Nord par des dômes-coulées chevauchant un linéament orienté SW-NE. Le Nevado Hualca-Hualca est également un grand strato-volcan très proche au NNW du complexe précédent mais davantage érodé. Ces appareils atteignent les altitudes respectives de $6280 \mathrm{~m}$ (Ampato), $6040 \mathrm{~m}$ (Sabancaya) et $6025 \mathrm{~m}$ (Hualca Hualca) qui, de ce fait, dominent de 1500 à
2000 m l'Altiplano portant les tourbières et lacs périphériques. Les dômes et les coulées de lave du Nevado Sabancaya, qui surmontent le substratum volcanique daté de 0,6 Ma environ (Gerbe \& Thouret, 2004), couvrent une superficie de $70 \mathrm{~km}^{2}$ environ, et occupent un volume approximatif de $25 \mathrm{~km}^{3}$. Au moins dix coulées de lave à blocs, de composition andésitique à dacitique subalcaline, et d'âge holocène, se sont épanchées sur une distance de $8 \mathrm{~km}$ vers le NW et le SE (fig. 2). Une coulée à blocs située sur le flanc NW du volcan repose sur une tourbe dont l'âge ${ }^{14} \mathrm{C}$ est d'environ 5400 ans BP (Lamadon, 1999). Des dépôts de coulées pyroclastiques à blocs et cendre reposent en alternance avec des retombées subaériennes; ils sont observés jusqu'à $9 \mathrm{~km}$ du sommet et sont attribués à l'activité explosive du Nevado Sabancaya ou de l'Ampato durant le Pléistocène supérieur.

Les tourbières et les salars (lagunes salées), situées sur les piémonts et dans les vallées glaciaires autour des strato-volcans cités plus haut sont des pièges sédimentaires qui ont pu enregistrer, entre autres, des retombées volcaniques (téphras) tant d'origine locale

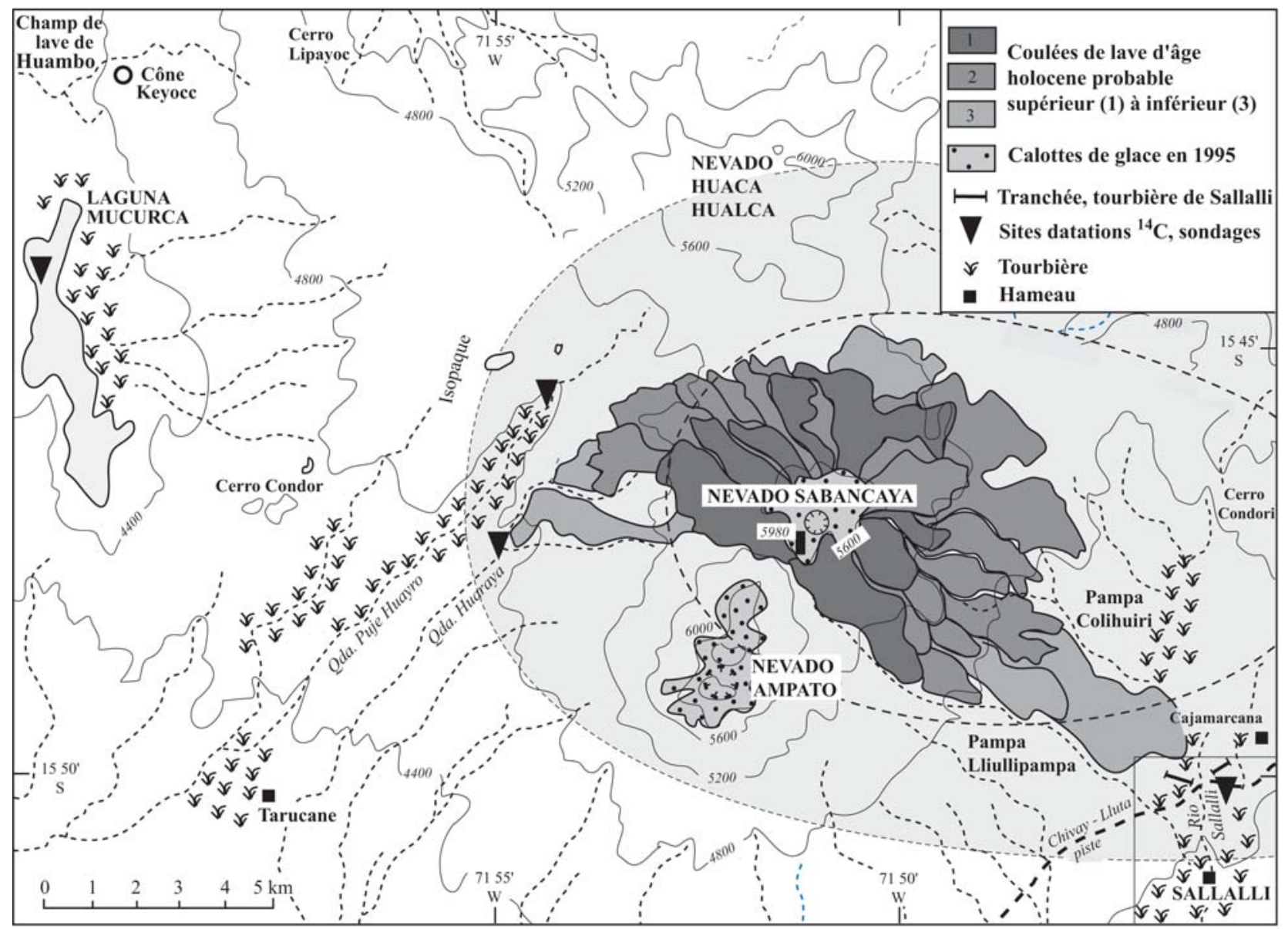

Fig. 2 : Nevados Ampato, Sabancaya et Hulca-Hualca ; la tourbière de Sallalli-Cajamarcana et le lac salé de Mucurca, ainsi que le canyon du Río Colca.

Le lobe des retombées de l'éruption récente du Sabancaya (1990-1998) est représenté par deux isopaques. Dans le coin inférieur droit de la figure, le rectangle délimite la tourbière, dont les sites étudiés sont représentés en détail à la figure 2B.

Fig. 2: Nevados (ice-clad volcanoes) Ampato, Sabancaya and Hualca Hualca; peat bog of Sallalli-Cajamarca and Mucurca brine lake (salar), as well as the canyon of the Río Colca river. The lobe of tephra dispersed during the last eruption of Nevado Sabancaya (1990-1998) is schematically shown by two isopachs. In the lower right corner, a rectangle outlines the peat bog in which sites for peat cores and ${ }^{14} C$ dating are shown in fig. $2 B$. 
que distale, comme l'illustrent le salar de la Laguna Salinas à $40 \mathrm{~km}$ à l'est d'Arequipa (Juvigné et al., 1997), et les grands salars de Bolivie (Risacher \& Fritz, 1991 ; Risacher, 1992). Ainsi avons-nous recherché des téphras, d'une part dans des tourbières sur le piémont oriental de l'Ampato et du Sabancaya (Sallalli et Cajamarcana), et d'autre part sur le piémont NW dans la Laguna Mucurca. De tels sites sont nécessairement postérieurs à la déglaciation des piémonts. La présente étude chronostratigraphique ne pouvait donc remonter au-delà de 15000 ans (Juvigné et al., 1997 ; Thouret et $a l ., 2002 \mathrm{a})$, voire 21000 ans dans la mesure où, sur la base de nouvelles datations par cosmonucléides, le maximum glaciaire a été daté dans le sud du Pérou à 34000 ans BP et le début du retrait à 21000 ans BP (Smith et al., 2005 ; Mark et al., 2004). Cependant, ces âges plus anciens ont été obtenus sur des moraines dans la Cordillère orientale, dont l'environnement climatique est et était différent de celui de la Cordillère occidentale, probablement moins humide.

\section{3 - ACTIVITÉ VOLCANIQUE RÉGIONALE PENDANT L'HOLOCÈNE ET LA PÉRIODE HISTORIQUE}

Le Nevado Sabancaya est le seul volcan du massif de l'Ampato-Sabancaya-Hualca qui a une activité historique connue ; il domine directement les piémonts étudiés ici. Thouret et al. (1994) ont rassemblé les données relatives à l'activité volcanique post-glaciaire dans ce massif ; elle est limitée en principe à celle du Nevado Sabancaya et résumée ci-après.

A des moments encore indéterminés de l'Holocène, le Sabancaya a construit deux dômes-coulées, avec de volumineuses coulées de lave à blocs de composition andésitique puis dacitique, et étalées sur plus de $40 \mathrm{~km}^{2}$ (Thouret et al., 1994). Des dépôts, les uns de retombées subaériennes, et les autres de coulées de scories et lapilli ponceux, ont également été observés dans des affleurements de sédiments volcanoclastiques postglaciaires.

Sur la base de la présence d'une lamine de cendre volcanique reconnue dans une tourbière du piémont oriental du Nevado Sabancaya, ce volcan aurait eu une éruption phréatomagmatique vers 8500 ans $\mathrm{BP}$ (Juvigné et al., 1998), soit environ 8000 cal BC.

Au pied du Nevado Sabancaya, une coulée de lave à blocs s'est étalée en bordure de la dépression à un moment encore indéterminé de l'Holocène. Quelques chroniques datant du XVIII siècle mentionnent deux phases d'activité de ce volcan, d'abord en 1752, puis en 1784-85 (Travada y Córdova, 1958). Par la suite, de 1785 à 1986, une activité fumerollienne faible et très sporadique a été relatée.

En novembre 1986, l'activité fumerollienne s'est accentuée. Des fractures se sont ouvertes, la glace s'est crevassée et un cratère s'est ouvert dans la glace, puis dans les laves du dôme-coulée septentrional. L'éruption du Nevado Sabancaya a été presque continue, mais modérée, de mai 1990 à 1998 . Cette éruption a mis fin à une phase de repos de 200 ans environ.

Entre mai 1990 et décembre 1994, l'activité du Sabancaya a adopté un caractère explosif, particulièrement intense lors de la crise du 28 mai au 5 juin 1990. Pendant ces neuf jours, un des panaches s'éleva jusqu'à une hauteur de $5000 \mathrm{~m}$ au-dessus du cratère, et ses retombées cendreuses furent retrouvées jusqu'à environ $20 \mathrm{~km}$ vers l'est du volcan (Thouret et al., 1994). L'activité vulcanienne modérée entre 1990 et 1994 a consisté en une série de brèves explosions séparées par des intervalles de repos de 15 à 60 minutes. Elles produisirent des colonnes éruptives hautes de 0,5 à $3 \mathrm{~km}$, intercalées dans de longues périodes de dégazage. Le matériau juvénile rassemble des fragments de lave non altérés, noirs et vitreux, de composition andésitique à dacitique (Thouret et al., 1994, 1995). Bien que le volume apparent de téphra émis ait été faible $\left(0,025 \mathrm{~km}^{3}\right)$, les retombées furent assez largement dispersées sur près de $250 \mathrm{~km}^{2}$.

Après 1994, l'activité s'est poursuivie de façon plus faible, plus sporadique et essentiellement phréatique. La nature des projections du Nevado Sabancaya a changé au cours du temps. Au départ, la part de xénolithes était très élevée (85-90\% en octobre 1990) à la faveur de l'ouverture de la cheminée, puis elle a diminué (40$50 \%$ en décembre 1992) au profit de fragments de magma juvénile jusqu'en 1994. Dans l'ensemble, le magma juvénile a une composition d'andésite potassique (Gerbe \& Thouret, 2004).

Un échantillon comprenant l'ensemble de ces retombées a été prélevé dans la tourbière de Sallalli, et il a été examiné dans le cadre du présent travail où il va être question d'identifier des téphras de composition hétérogène. La distribution granulométrique est unimodale avec un mode entre $125 \mu \mathrm{m}$ et $250 \mu \mathrm{m}$; le plus gros élément atteint $16 \mathrm{~mm}$, la fraction poussiéreuse $(<63 \mu \mathrm{m})$ représente $12 \%$ de l'ensemble. On y trouve un large éventail de composants : scories gris foncé, débris de coulée de lave gris foncé, traces de verres bruns ou incolores, minéraux felsiques (essentiellement feldspaths et très secondairement quartz), minéraux mafiques (micas bruns, clinopyroxènes, orthopyroxènes et amphiboles). Une telle variété atteste un mélange des différents matériaux accumulés dans et sur le cône, et repris ou remobilisés lors de l'ouverture et de l'élargissement de la cheminée.

\section{4 - LA TOURBIÈRE DE SALLALLI, RÉSULTATS}

La tourbière de Sallalli-Cajamarcana est située sur le piémont oriental du Nevado Sabancaya $\left(16^{\circ} 45^{\prime} \mathrm{S}\right.$, $71^{\circ} 48^{\prime} \mathrm{W}$ ) à une altitude de $4400 \mathrm{~m}$ (fig. 2 et 3). Elle occupe une dépression entourée par des cordons morainiques, et affectée de nombreuses dépressions thermokarstiques (kettle holes) développées dans des dépôts glaciaires et fluvio-glaciaires. Le Rio Sallalli draine la dépression et se jette dans le Rio Parcomayo sur le piémont sud du massif étudié. 

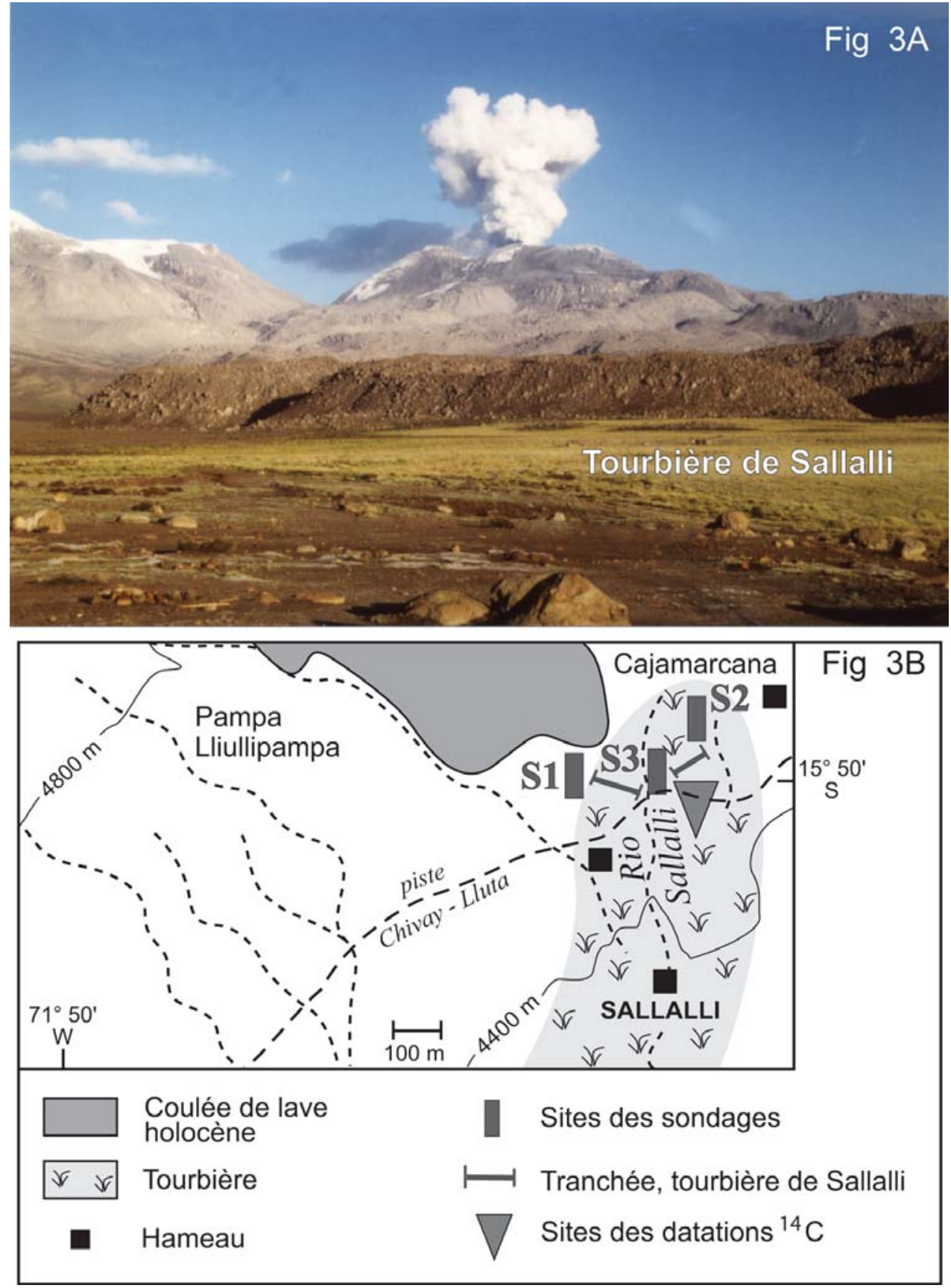

Fig. 3 : A. Le Sabancaya en éruption en mai 1996 (photo J.-C. Thouret) et la tourbière de Sallalli envahie par des coulées de lave à blocs de l'Holocène supérieur ; B. Position des sites étudiés dans la tourbière de Sallalli. Ce secteur est localisé dans la figure 2.

Fig. 3: A. Nevado Sabancaya erupting in May 1996 (photograph JC Thouret) and peat bog of Sallalli, which has been partly covered by block-lava flows sometime during the upper Holocene. B. Location of studied sites in the peat bog. This area is located in Fig. 2.

Un affleurement tourbeux, correspondant à la berge de rive gauche du Rio Sallalli (fig. 4 : Coupe A), a été étudié antérieurement (Juvigné et al., 1998). Il couvre essentiellement le début de l'Holocène entre $9650 \pm$ 170 aBP (Lv-2110, soit 8400-9250 cal BC) et $8330 \pm$ 120 aBP (Lv-2121, soit 7080-7580 cal BC ; âges calibrés avec le modèle de Stuivert et al., 1998). Cet âge le plus ancien obtenu à la base des séquences étudiées fournit une limite chronologique minimale pour la déglaciation du piémont étudié. L'affleurement tourbeux comprend quatre niveaux sableux dont un a été identifié comme téphra (Juvigné et al., 1997) correspondant à une éruption phréatomagmatique du
Sabancaya, qui aurait eu lieu vers $8520 \pm 80$ aBP (Lv2107), soit entre 7420 et 7750 cal BC. Nos nouvelles recherches ont porté sur une fouille en tranchée et des carottes extraites au moyen d'une sonde russe (fig. 3A).

\section{1 - FOUILLE EN TRANCHÉE}

Une tranchée d'une centaine de mètres de longueur environ a été réalisée sur la rive droite du Rio Sallalli (fig. 4) et, sur le tronçon où le sable et gravier n'a pas été atteint, des sondages ont été exécutés sur le fond 


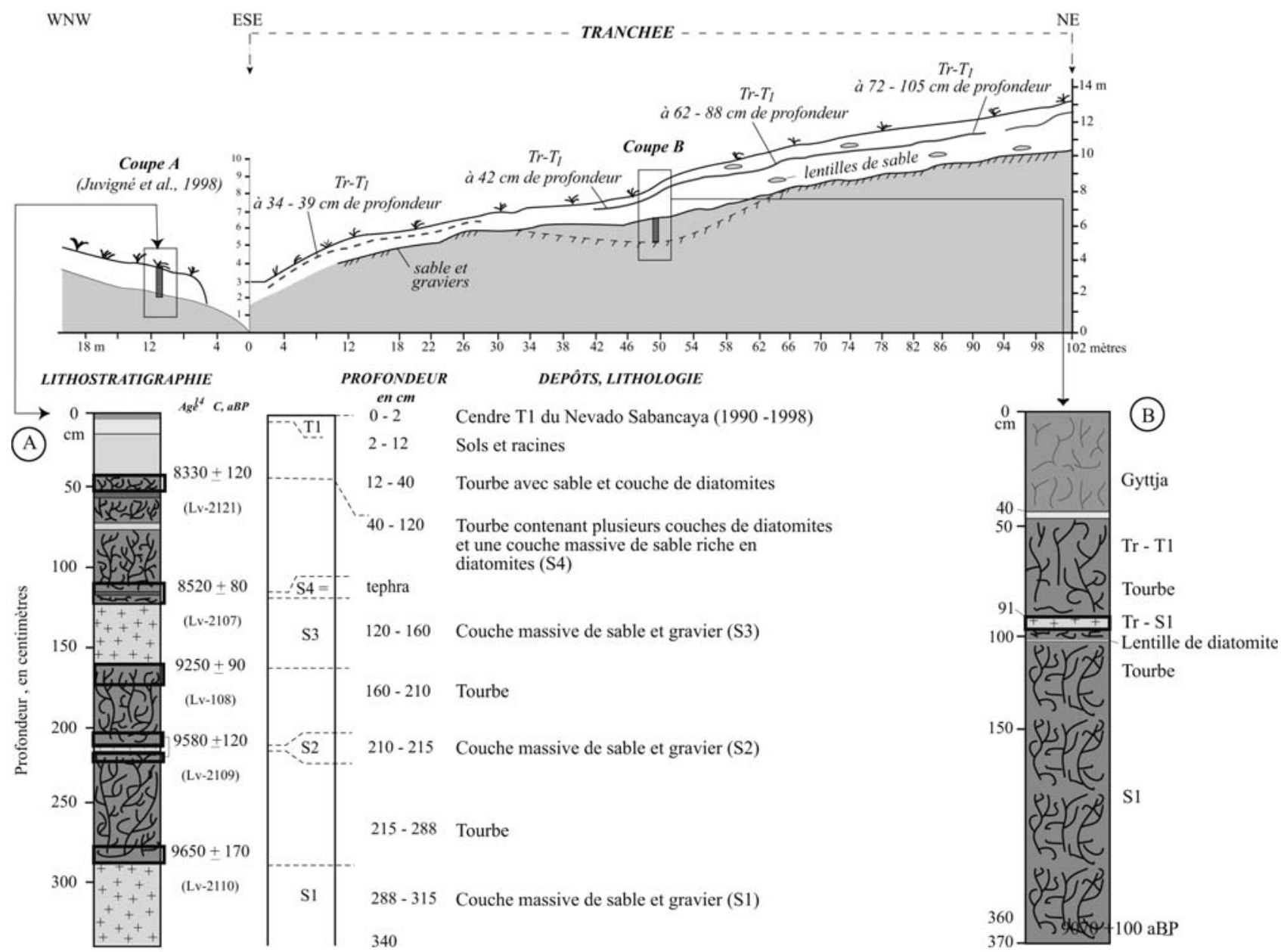

Fig. 4 : Coupes dans le vallon du Rio Sallalli. Coupe A, étudiée par Juvigné et al. (1998) ; Coupe B, paroi de la tranchée (de 0 à 150 cm), prolongée par une carotte de sondage (S1).

Fig. 4: Cross sections in the small-scale Rio Sallalli valley: cross section A has been studied by Juvigné et al. (1998); cross section B is the 0 to $150 \mathrm{~cm}$ deep trench wall, which has been deepened by the S1 core drilled in the peat bog.

pour mesurer l'épaisseur totale de la tourbe, qui atteint localement $370 \mathrm{~cm}$ sous la surface. C'est à cet endroit de plus forte épaisseur de tourbe qu'à partir du fond un carottage a été effectué (fig. 4 : Coupe C + sondage S1).

\subsection{1 - Un téphra}

Sur la paroi de la tranchée, il existe des lentilles, dont une d'une quarantaine de mètres de longueur, pouvant atteindre $3 \mathrm{~cm}$ d'épaisseur et prolongée par d'autres occurrences éparses ; elles se situent à une profondeur variant entre 34 et $105 \mathrm{~cm}$. La taille de l'élément le plus gros est de $2 \mathrm{~mm}$. La détermination au microscope polarisant dans la classe de 105 à $420 \mu \mathrm{m}$ fait apparaître une majorité de microponces et de verres volcaniques vacuolaires incolores. Le verre est de composition rhyolithique à tendance potassique (voir tab. 2 et fig. 8). La fraction cristalline se répartit en $80 \%$ de minéraux felsiques (essentiellement des feldspaths) et $20 \%$ de minéraux mafiques (dont $2 \%$ de mica brun). Dans un échantillon de minéraux denses séparés dans le bromoforme, les minéraux ferromagnésiens (autres que les micas bruns) consistent en clinopyroxènes vert à brun clair $(57 \%)$, orthopyroxènes $(22 \%)$ et amphiboles $(21 \%)$.

Les feldspaths consistent essentiellement en oligoclase potassique et andésine et secondairement en labrador (tab. 3). Dans la classification de Morimoto (1989), l'orthopyroxène est de l'enstatite (hypersthène) et les clinopyroxènes se répartissent de part et d'autre de la limite des champs de l'augite et du diopside (tab. 3). Dans la classification de Leake et al. (1997), les amphiboles sont calciques du type magnesio-hornblende. Il s'agit d'un téphra provenant d'une éruption plinienne ; nous l'appelons Tr-T1 (Tranchée, Téphra 1).

Un échantillon de tourbe de $3 \mathrm{~cm}$ d'épaisseur comprenant le téphra a été daté par le ${ }^{14} \mathrm{C}$ et a fourni un âge de $300 \pm 50$ aBP (Beta-126965), soit entre 1460 et 1670 cal AD.

Dans la mesure où au moment de la retombée le téphra existait nécessairement en nappe continue, la structure lenticulaire atteste le remaniement au moins partiel du téphra. Dans ce contexte, seul le vent peut avoir été responsable de ce remaniement. 


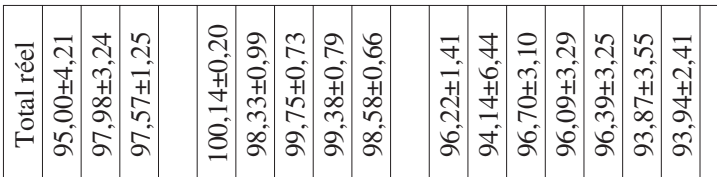

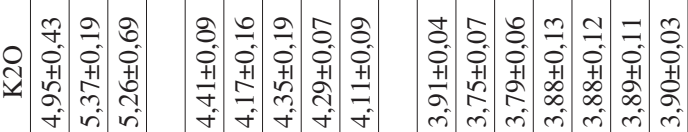

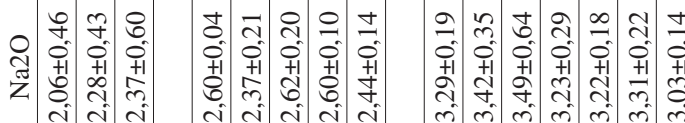

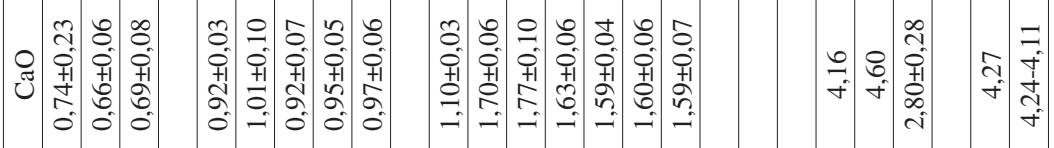

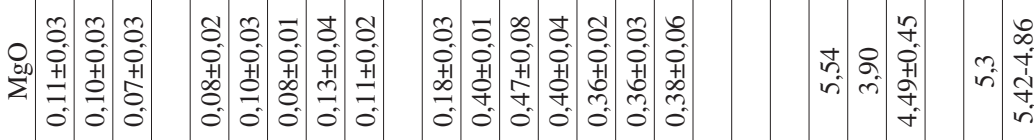

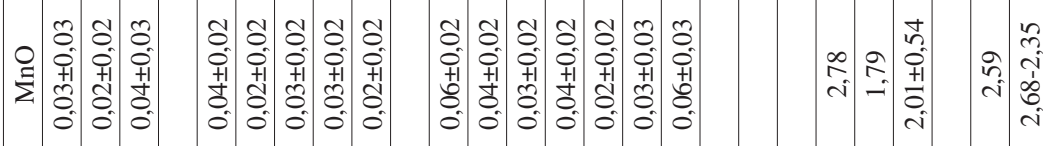

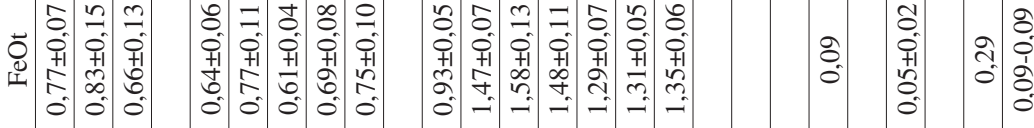

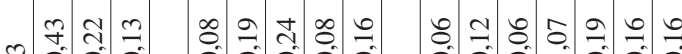

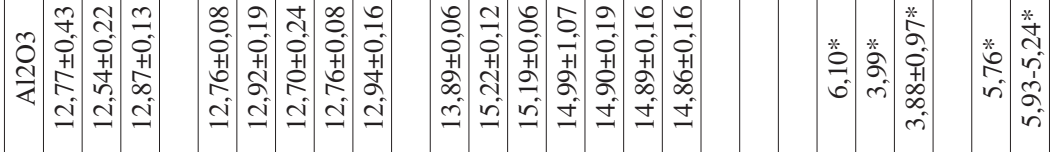

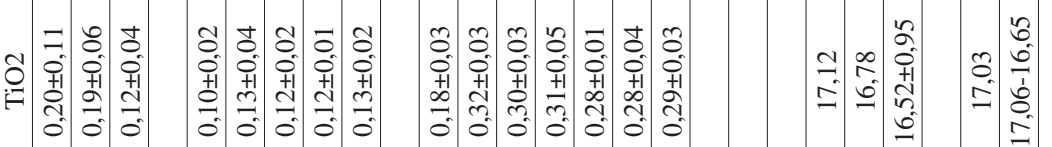

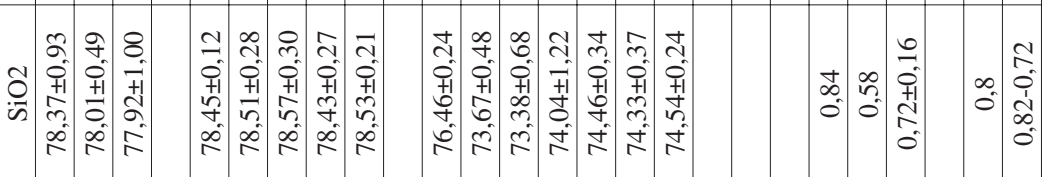

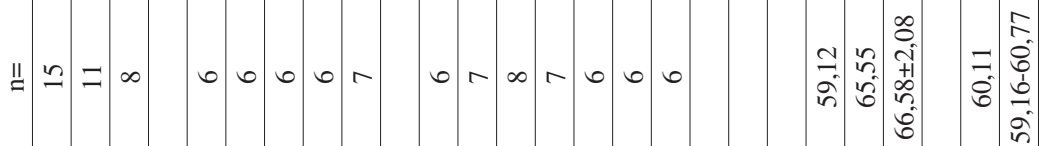

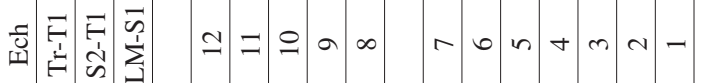

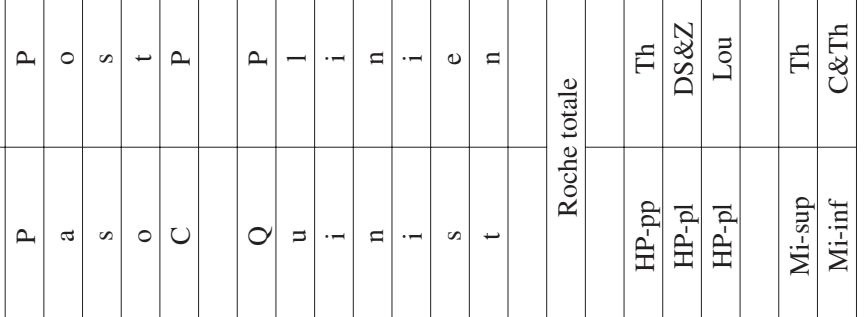

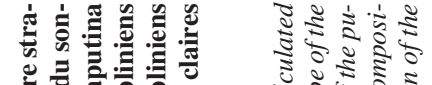

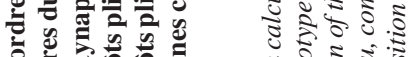

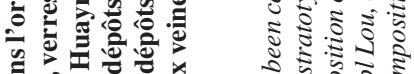

की

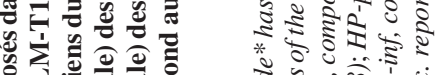

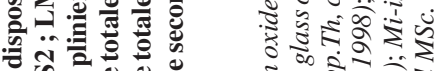

\%

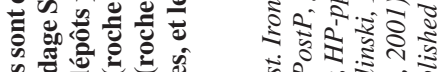

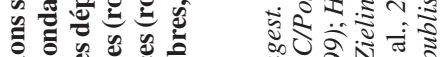

을

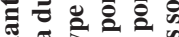

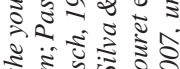

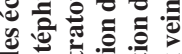

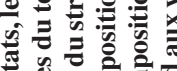

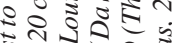

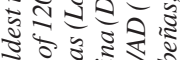

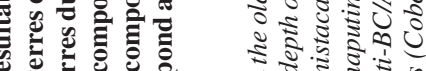

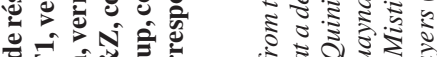

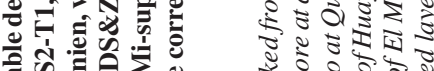

ह

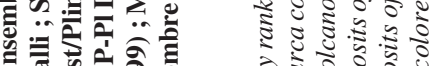

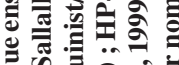

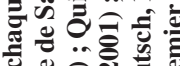

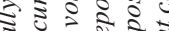

8.

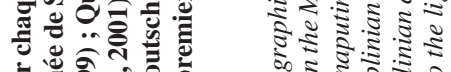

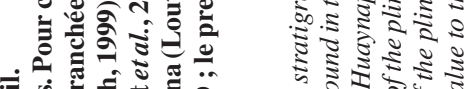

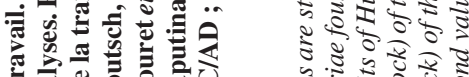

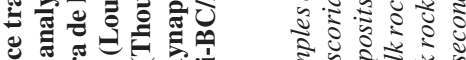

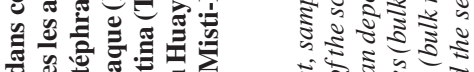

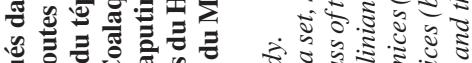

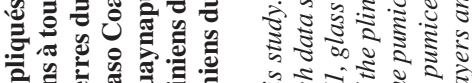

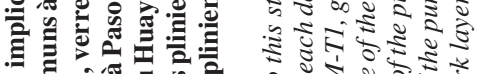

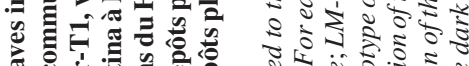

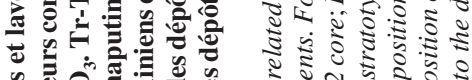

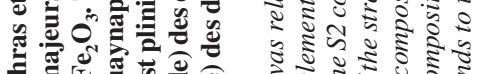

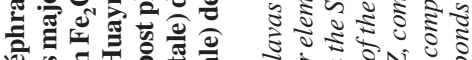

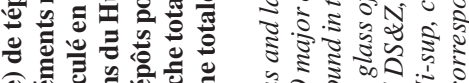

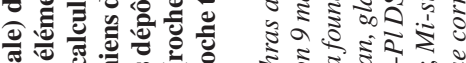

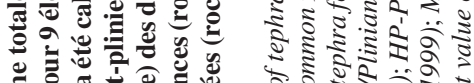

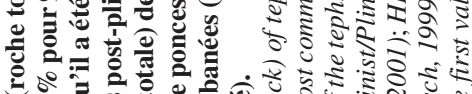

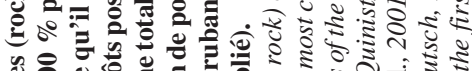

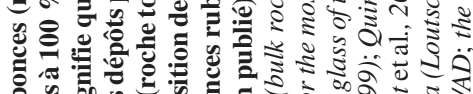

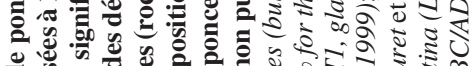

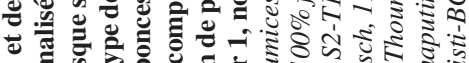

कू

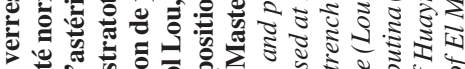

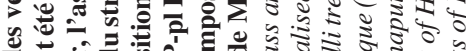

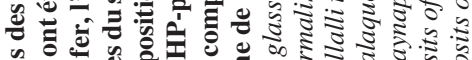

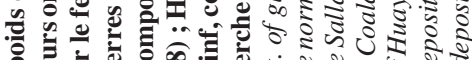

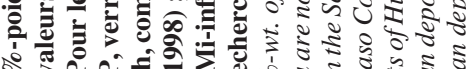

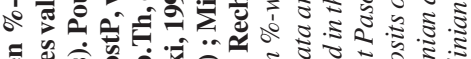
च

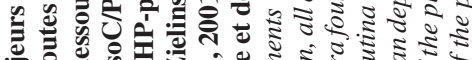

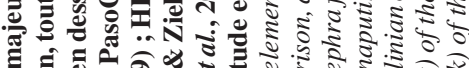

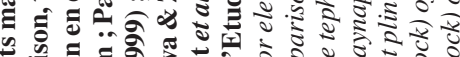

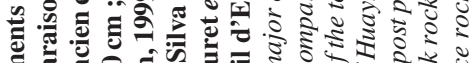

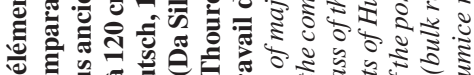

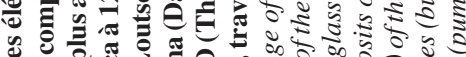

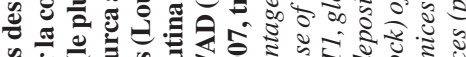

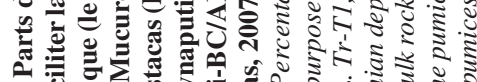

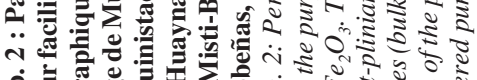
An 


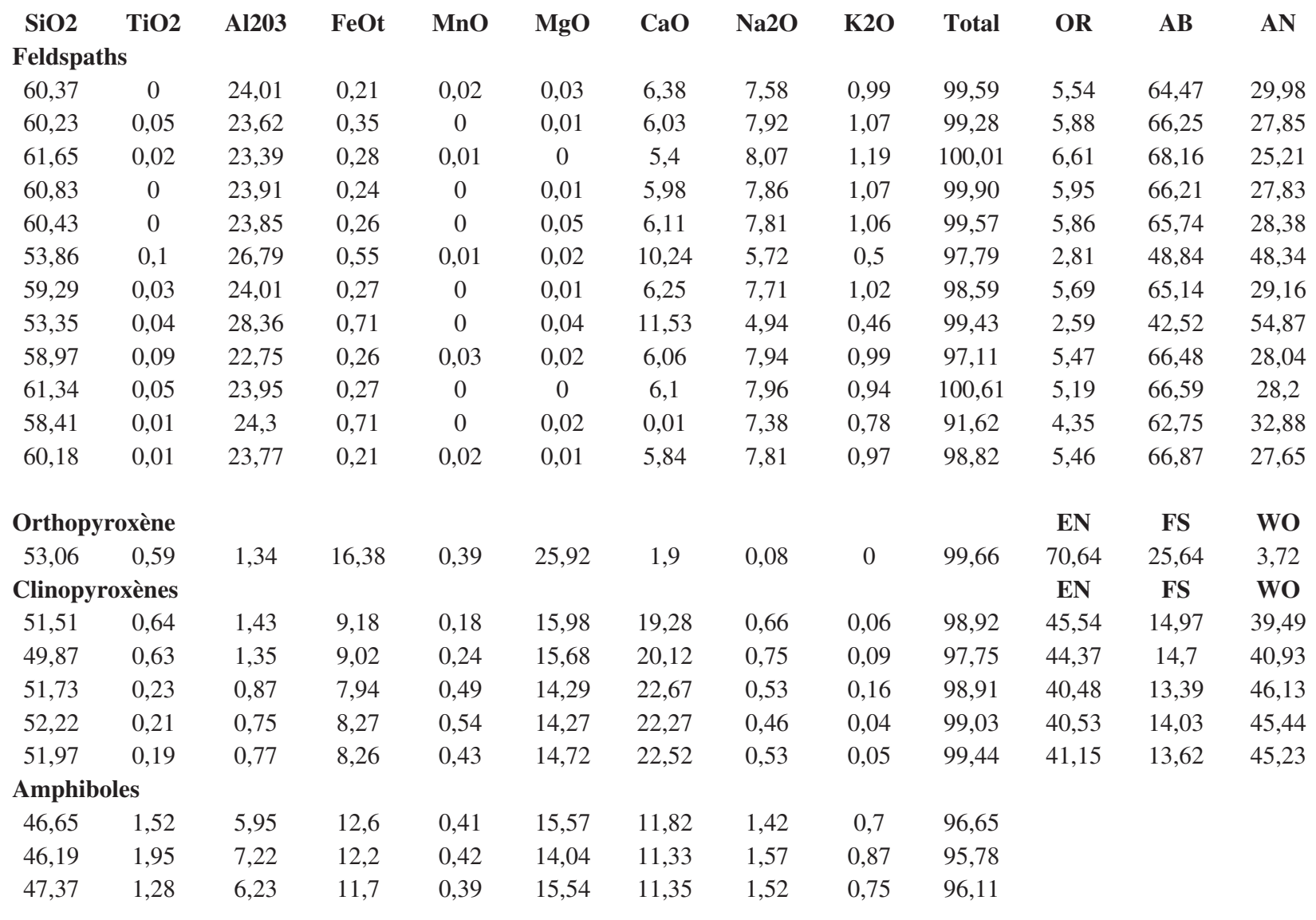

Tab. 3 : Composition chimique de minéraux du téphra Tr-T1 de la tranchée de Sallalli.

Tab. 3: Geochemical composition of the minerals of the Tr-T1 tephra found in the trench dug out in the Sallalli peat bog.

\subsection{2 - Quelques autres lentilles minérales}

Cinq niveaux de lentilles minérales de faible extension (décimètre) ont été examinés.

L'un d'entre eux, situé une cinquantaine de centimètres sous le téphra, est un sable de composition hétérogène dont les grains essentiels sont des scories gris foncé, des débris de coulée de lave gris foncé, des feldspaths et des minéraux mafiques divers: micas bruns, clinopyroxènes, hypersthènes et amphiboles. Par comparaison avec le téphra récent du Sabancaya, ce sable peut être un téphra de retombée phréatomagmatique; nous l'appelons Tr-S1 (Tranchée, Sable 1). Quatre autres niveaux consistent essentiellement en diatomite.

\section{2 - SONDAGE S1}

A l'endroit de la coupe B, depuis le fond de la tranchée $(150 \mathrm{~cm})$ jusqu'à la base du sondage $\mathrm{S} 1$ (à $370 \mathrm{~cm}$ ), la tourbe est pratiquement pure jusqu'au refus sur du matériau minéral meuble. La tourbe de base (360-370 cm) a été datée de $9070 \pm 100$ aBP (Hv24659), soit entre 8125 et 8020 cal BC. Compte tenu de cet âge, on peut admettre que le matériau minéral sousjacent qui a provoqué le refus de la sonde russe devrait être l'épais téphra phrétomagmatique connu dans la coupe A, sur la berge toute proche du Rio Sallalli (Juvigné et al., 1997).

\section{3 - SONDAGE S2}

La carotte S2 a été prélevée sur le versant de rive droite, environ $400 \mathrm{~m}$ à l'amont de la tranchée (fig. 3B) et à proximité de la limite de la tourbière. Les résultats des analyses sont représentés à la figure 5.

De la surface jusque $165 \mathrm{~cm}$, la tourbe est d'apparence pure et l'âge du segment de 145 à $165 \mathrm{~cm}$ a été daté de $2050 \pm 70 \mathrm{aBP}(\mathrm{Lv}-2184)$ soit entre 210 cal BC et $130 \mathrm{cal} \mathrm{AD.}$

Sous la tourbe, la difficulté d'enfoncer la sonde a provoqué la perturbation des sédiments, et la stratigraphie présentée constitue un essai de reconstitution :

a) de 165 à $185 \mathrm{~cm}$, un sable gravillonneux de distribution granulométrique bimodale dont le plus gros grain atteint $32 \mathrm{~mm}$; nous l'appelons S2-S1 (Sondage 2- Sable 1).

b) de 185 à $195 \mathrm{~cm}$, sable de composition hétérogène, riche en verres vacuolaires incolores. Il s'agit de $87 \%$ de cendre (mode principal d'environ $250 \mu \mathrm{m}$ ) avec une faible fraction poussiéreuse $(13 \%)$. La composition chimique des verres correspond à de la rhyolithe potassique (voir plus loin, fig. 8). Dans les frottis de minéraux denses entre 105 et $420 \mu \mathrm{m}$, on trouve $63 \%$ de clinopyroxènes, $18 \%$ d'orthopyroxènes (hypersthène) et $19 \%$ d'amphiboles. Il s'agit d'un téphra provenant d'une éruption plinienne; nous 1'appelons S2-T1 (Sondage 2, Téphra 1). 
(A)

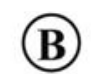

DISTRIBUTION GRANULOMETRIQUE

(C)

MINERAUX LOURDS

\section{SONDAGE S2, SALLALLI}

LITHOSTATIGRAPHIE
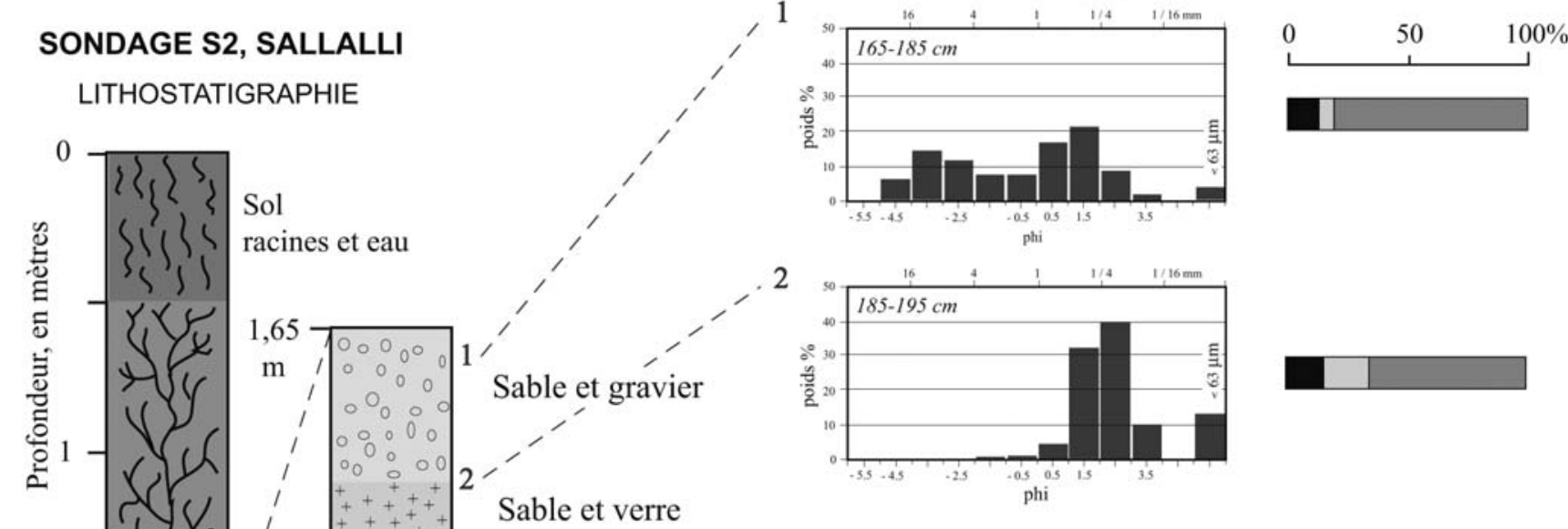

Sable et verre
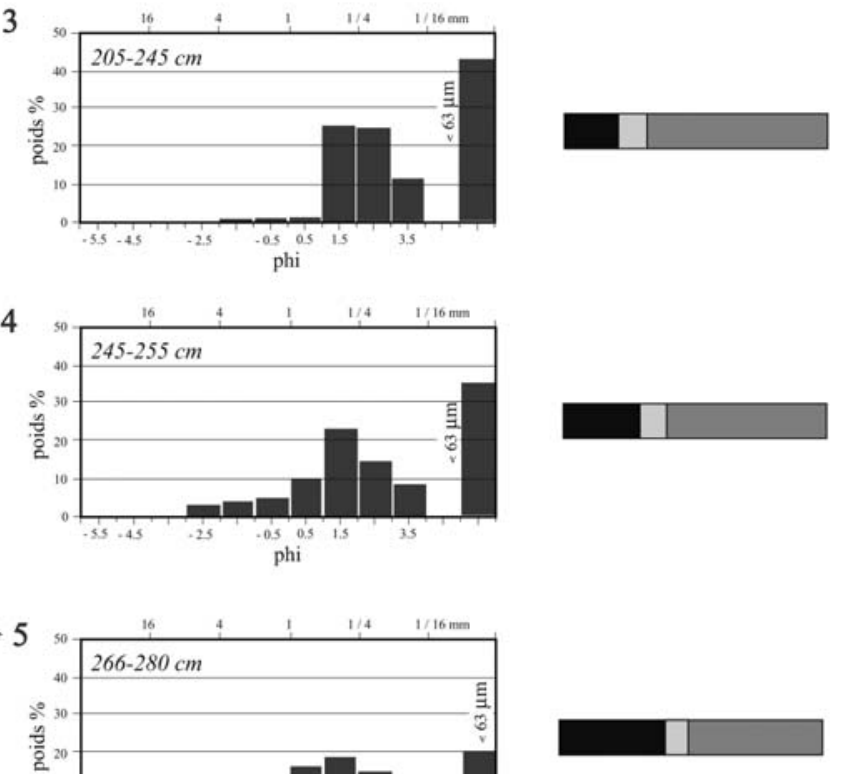

Sable grossier stratifié Lentilles de limons

Diatomites
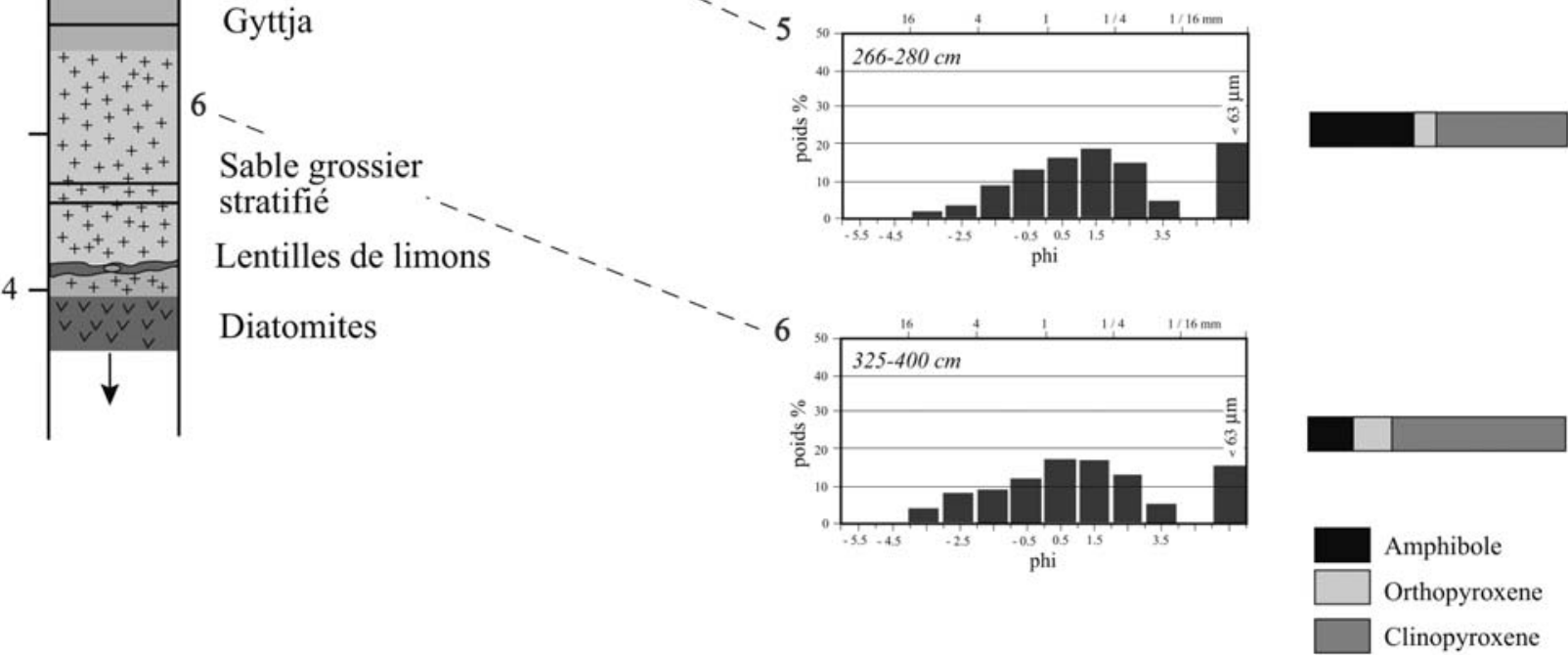

Fig. 5 : A. Lithostratigraphie de la carotte du sondage S2 ; B. Distribution granulométrique des couches minérales ; C. Minéraux denses de plus de plus de $105 \mu \mathrm{m}$ des mêmes couches.

Fig. 5: A. Lithostratigraphy of the S2 core; B. Grain size distribution of the mineral layers; C. Heavy minerals exceeding $105 \mu$ m in these mineral layers.

c) De 195 à $205 \mathrm{~cm}$, une couche de tourbe a donné un âge de $2370 \pm 90 \mathrm{aBP}(\mathrm{GrN}-25586$, soit entre $800 \mathrm{cal}$ $\mathrm{BC}$ et $200 \mathrm{cal} \mathrm{AD})$.

De 205 à $325 \mathrm{~cm}$, l'essentiel de la masse a une texture argileuse et contient de nombreuses frustules de diatomées et de la matière organique végétale diffuse (gyttja). Des niveaux sableux à gravillonneux sont intercalés dans ce gyttja : de 205 à $210 \mathrm{~cm}$, de 245 à $255 \mathrm{~cm}$, de 265 à $280 \mathrm{~cm}$. De 325 à $400 \mathrm{~cm}$, du sable grossier apparemment stratifié (voir plus haut : perturbation par sondage) est présent dans la partie inférieure du gyttja. Chacune de ces couches sableuses contient une fraction fine qui peut être en partie héritée du gyttja hôte, qui a pu y pénétrer en profitant de la porosité. La fraction sablo-gravillonneuse de ces couches est constituée d'une grande variété d'éléments : débris de coulées de lave, scories basaltiques, cristaux variés, felsiques (feldspaths et quartz) et 
(A)

\section{LITHOSTRATIGRAPHIE}

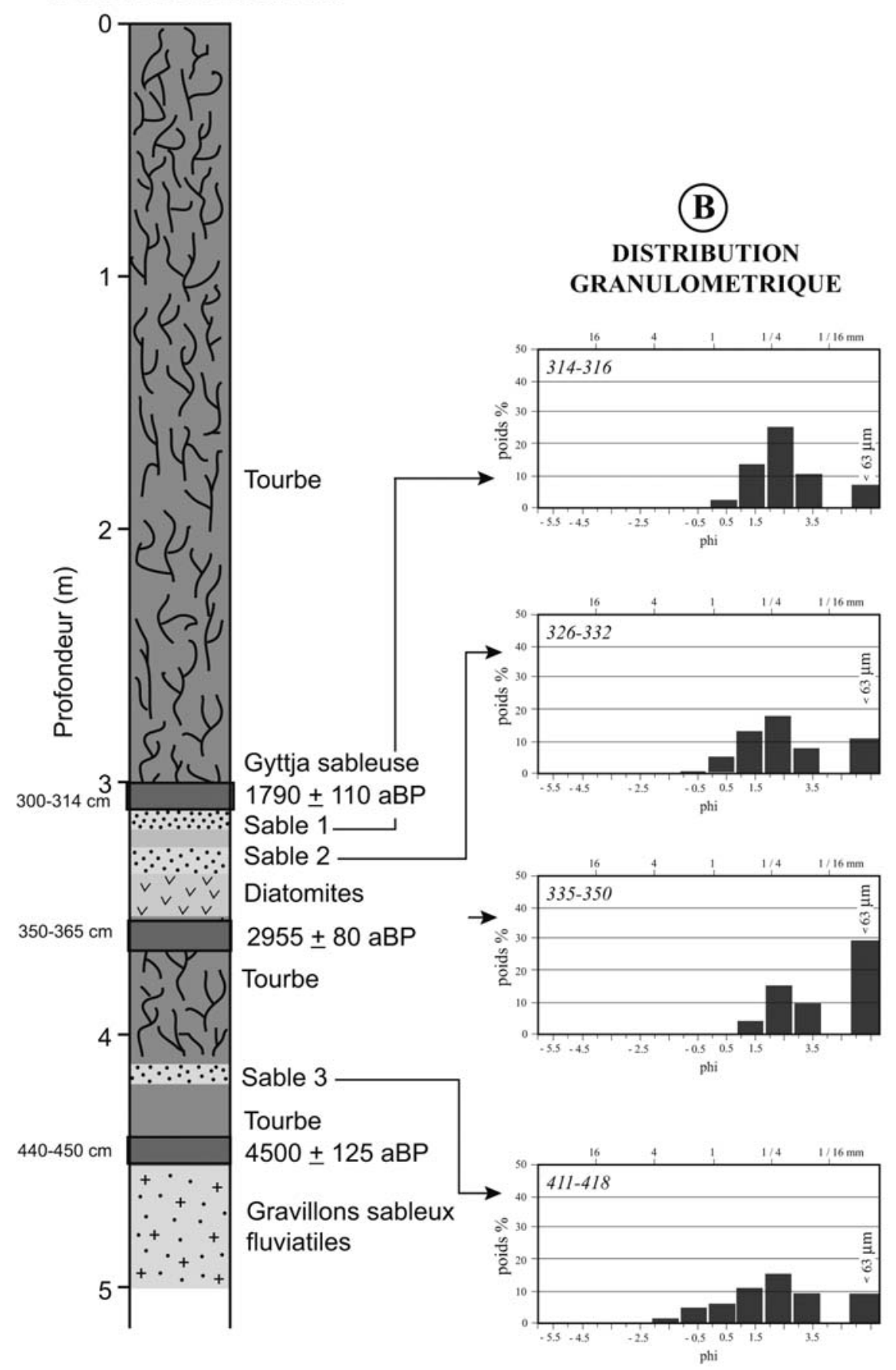

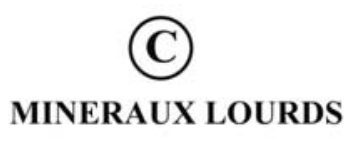
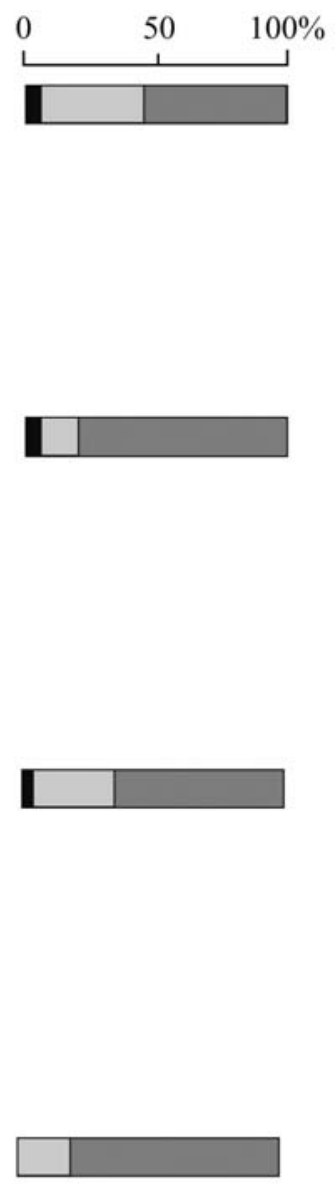

Amphibole

Orthopyroxene

Clinopyroxene

Fig. 6 : A. Lithostratigraphie de la carotte du sondage S3 ; B. Distribution granulométrique des couches minérales ; C. Minéraux denses excédant $105 \mu \mathrm{m}$ des mêmes couches $(C)$.

Fig. 6: A. Lithostratigraphy of the S3 core; B. Grain size distribution of the mineral layers; C. Heavy minerals exceeding $105 \mu$ m in these mineral layers.

mafiques (clinopyroxènes, orthopyroxènes, micas bruns, amphiboles). Par comparaison avec le téphra récent du Sabancaya, ces sables peuvent être des téphras de retombées phréatomagmatiques; nous les appelons respectivement : (i) $205-210 \mathrm{~cm}=\mathrm{S} 2-\mathrm{S} 2$ (Sondage 2- Sable 2); (ii) 245-255 cm = S2-S3 (Sondage 2- Sable 3); (iii) 266-280 cm = S2-S4 (Sondage 2- Sable 4); (iv) 325-340 $\mathrm{cm}=$ S2-S5 (Sondage 2- Sable 5). Par comparaison avec le téphra récent du Sabancaya, ces sables pourraient être des produits d'éruptions phréatomagmatiques.

\section{5 - SONDAGE S3}

Le point de prélèvement de la carotte $\mathrm{S} 3$ se situe dans une dépression thermokarstique (kettle hole) à environ $500 \mathrm{~m}$ au nord de la tranchée du Rio Sallalli 
(fig. 3B). Les résultats des analyses sont représentés à la figure 6.

De la surface jusqu'à $450 \mathrm{~cm}$ de profondeur, on recoupe essentiellement de la tourbe à macrorestes, dans laquelle sont intercalées trois couches sableuses (314-316 cm ; 326-332 cm ; 411-418 cm) et une diatomite $(335-350 \mathrm{~cm})$. La sonde a été bloquée à $5 \mathrm{~m}$ de profondeur dans une couche de gravillon d'au moins $50 \mathrm{~cm}$ d'épaisseur. Ce gravillon sableux de base (450$500 \mathrm{~cm}$ ), par la granularité et la variété de ses éléments, devrait avoir été déposé par le ruissellement.

Les couches sableuses (sables 1, 2 et 3 ) sont toutes de couleur gris foncé à noire. Leurs bords inférieurs et supérieurs sont nets. Leurs granularités respectives présentent un seul mode entre 125 et $250 \mu \mathrm{m}$, assorti d'un bon classement. La part de la fraction limonoargileuse est comprise entre $8 \%$ et $10 \%$ (dont des diatomées). Du point de vue minéralogique, on y retrouve l'éventail classique propre à cette région volcanique : scories gris foncé, débris de coulée de lave gris foncé, traces de verres bruns ou incolores, minéraux felsiques (essentiellement feldspaths et très secondairement quartz), minéraux mafiques (micas bruns, clinopyroxènes, orthopyroxènes (hypersthène) et amphiboles). L'ensemble de ces caractéristiques, et notamment les limites supérieures et inférieures nettes ainsi que l'isolement dans la séquence, permet d'attribuer ces trois couches à des retombées d'éruptions phréatomagmatiques. Nous les appelons respectivement: (i) 314$316 \mathrm{~cm}, \mathrm{~S} 3-\mathrm{T} 1$ (= Sondage 3- Téphra 1) ; ( ii) 326$332 \mathrm{~cm}, \mathrm{~S} 3-\mathrm{T} 2$ (= Sondage 3- Téphra 2) ; (iii) 411418 cm S3-T3 (= Sondage 3- Téphra 3).

Trois échantillons de tourbe ont été datés : (1) de 300 à $314 \mathrm{~cm}: 1790 \pm 110 \mathrm{aBP}(\mathrm{Hv}-24660$, soit entre 115 et $395 \mathrm{cal} \mathrm{AD})$; (2) de 350 à $365 \mathrm{~cm}: 2955 \pm 80 \mathrm{aBP}(\mathrm{Hv}-$ 24662 , soit entre 1290 et 1015 cal BC) ; (3) de 440 à $450 \mathrm{~cm}: 4500 \pm 125 \mathrm{aBP}(\mathrm{Hv}-24661$, soit entre 3365 et 2920 cal BC). Par extrapolation linéaire et en décomptant l'épaisseur des trois téphras, les résultats des trois datations permettent de conférer les âges approximatifs suivants aux trois téphras: S3-T1: entre environ 150 cal AD et 100 cal BC; S3-T2, entre environ $150 \mathrm{cal}$ $\mathrm{BC}$ et $420 \mathrm{cal} \mathrm{BC}$; S3-T3, entre environ $2100 \mathrm{cal} \mathrm{BC}$ et 2500 cal BC.

\section{5 - LAGUNA MUCURCA, RÉSULTATS}

Le salar de la Laguna Mucurca est situé sur le piémont NW des volcans Ampato - Hualca Hualca $\left(15^{\circ} 42^{\prime} \mathrm{S}, 72^{\circ} 01^{\prime} \mathrm{W}\right)$ à $4300 \mathrm{~m}$ d'altitude et à proximité des cônes et des champs de lave de Huambo (fig. 2). Dans la partie septentrionale de la Laguna Mucurca, une carotte a été prélevée au bord du lac, à un moment (avril 1995) où le niveau était bas. Le site de sondage a été choisi le plus loin possible des dépôts de ruissellement, qui s'étalent en cônes de déjection au pied des versants en pente forte fermant la dépression. Les résultats des analyses sont représentés à la figure 7 .
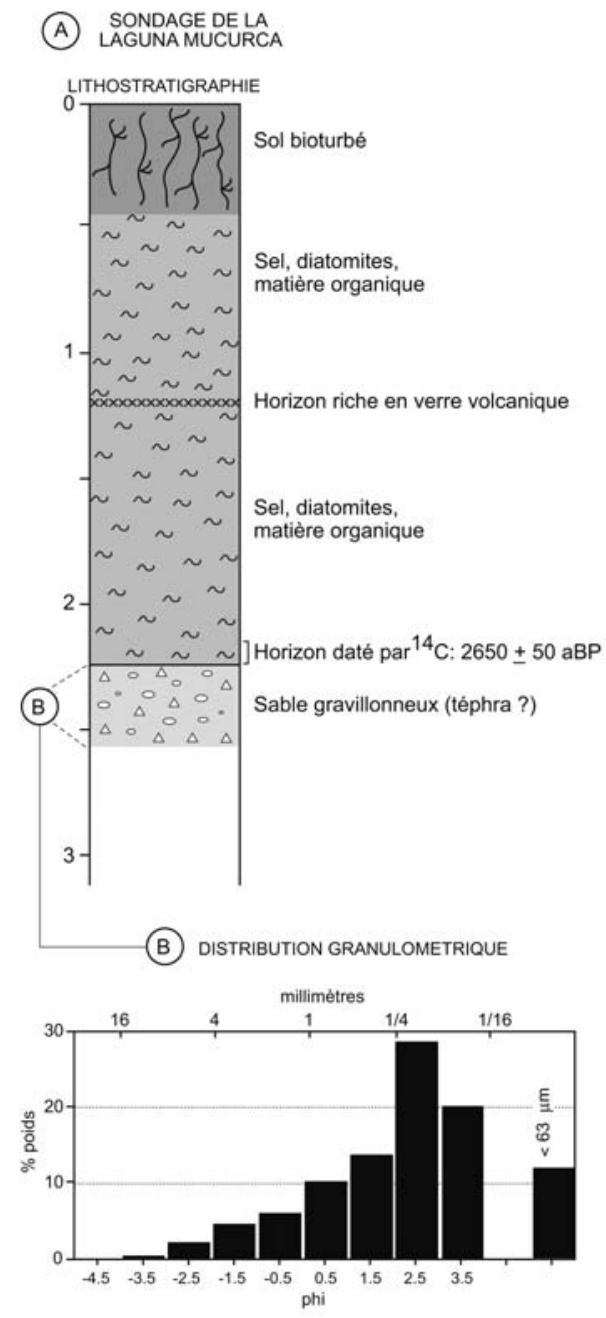

Fig. 7 : Lithostratigraphie de la carotte de la Laguna Mucurca ; A. Position du niveau riche en verre volcanique ; $B$. Distribution granulométrique du sable gravillonneux sous $225 \mathrm{~cm}$.

Fig. 7: Lithostratigraphy of the core drilled in the Laguna Mucurca; A. Location of the layer rich in volcanic glass; $B$. Grain size distribution of the gravel sand layer below $225 \mathrm{~cm}$ in depth.

Jusqu'à $225 \mathrm{~cm}$ de profondeur (fig. 7), la séquence consiste en un sédiment hôte limono-argileux riche en diatomées et matières organiques et contenant des cristaux de sel dans des proportions variables. La couleur d'ensemble est généralement vert olive avec des horizons plus foncés que d'autres en fonction de la teneur en matière organique. Aucune stratification n'est perceptible dans cette séquence. Un sol peu développé se présente dans l'horizon supérieur. A $225 \mathrm{~cm}$ de profondeur, il existe un contact brutal entre la formation décrite ci-dessus et une couche essentiellement sablogravillonneuse qui n'a pu être traversée entièrement, mais a néanmoins été prélevée sur $25 \mathrm{~cm}$ d'épaisseur. La tranche de 210 à $225 \mathrm{~cm}$ de dépôt lacustre surmontant immédiatement cette couche a donné un âge de $2650 \pm 50$ aBP $(\mathrm{GrN}-21575)$, soit entre 920 et $760 \mathrm{cal}$ BC. Le dépôt sablo-gravillonneux immédiatement sous-jacent devrait être de peu antérieur à cet âge. 


\section{1 - UN HORIZON RICHE EN VERRES VACUOLAIRES INCOLORES}

En l'absence de stratification visible dans la séquence jusqu'à $225 \mathrm{~cm}$ de profondeur, la recherche de verres volcaniques été réalisée au microscope. Pour ce faire, la carotte a été divisée en segments de $10 \mathrm{~cm}$ de hauteur. A $120 \mathrm{~cm}$ de profondeur, on trouve une quantité très élevée de verres vacuolaires incolores et de nombreux feldspaths enrobés de verre de composition rhyolithique et potassique (voir plus loin tab. 2 et fig. 8). Ces produits appartiennent donc à un téphra issu d'une retombée plinienne d'origine allochtone; nous 1'appelons LM-T1 (Laguna Mucurca- Téphra 1). La détermination de l'association minéralogique du téphra n'a pas été possible en raison, d'une part de la faible concentration de matériau téphrique, et d'autre part de la présence de minéraux volcaniques dans le sédiment hôte dans lequel le téphra est dispersé.

\section{2 - DESCRIPTION DU SABLE GRAVILLONNEUX $(225-250 \mathrm{~cm})$}

Le matériau est essentiellement sableux (75\%), secondairement gravillonneux (13\%) et limono-argileux ( $12 \%$, dont une part de diatomées). La distribution est unimodale avec un mode entre 125 et $250 \mu \mathrm{m}$. L'élément le plus gros atteint $16 \mathrm{~mm}$.

A la loupe binoculaire, on distingue : a) des phénocristaux sialiques libres, surtout des feldspaths ; b) des scories contenant des microcristaux; c) des débris de coulée de lave gris foncé, anguleux ou émoussés; d) des scories rougeâtres (faciès oxydé de cœur de cône) anguleuses ou émoussées ; e) quelques cristaux mafiques. Au microscope polarisant et dans la classe $105-420 \mu \mathrm{m}$, on trouve $90 \%$ de clinopyroxènes verts souvent automorphes, $5 \%$ d'orthopyroxènes (hypersthène) et $5 \%$ d'amphiboles de pléochroïsme vert à brun. Par comparaison avec le téphra récent du Sabancaya, ce sable peut être un téphra de retombée phréatomagmatique; nous l'appelons LM-S1 (Laguna Mucurca, Sable 1).

\section{6 - DISCUSSION}

Trois niveaux de téphra, chacun le plus récent dans leur site, sont caractérisés par la dominance de verres incolores vacuolaires de composition rhyolithique et potassique (tab. 2, fig. 8). Tous les téphras plus anciens ont la propriété commune d'avoir une texture très

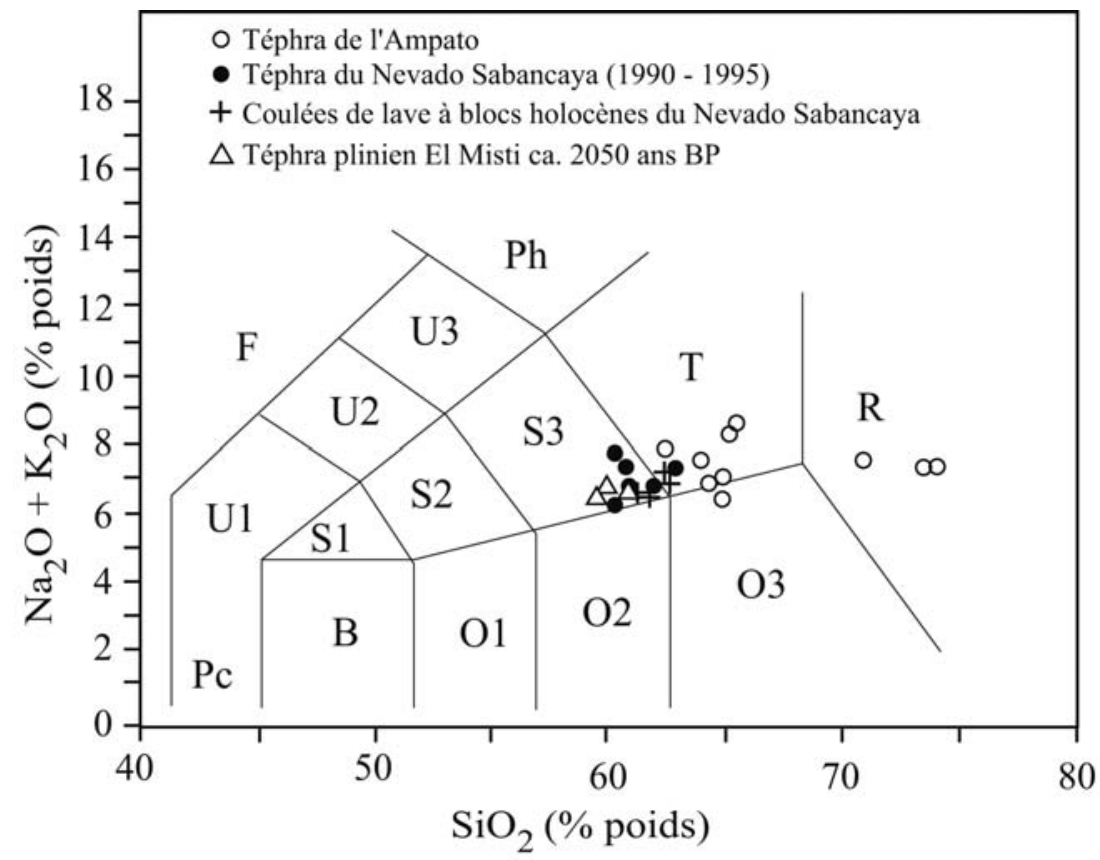

Fig. 8 : Détermination dans la classification TAS (Le Bas et al., 1986) des verres des sites de la tranchée de Sallalli et de la carotte de Mucurca, ainsi que de ceux des stratotypes des produits du Huaynaputina à Quinistacas (dépôts pliniens primaires) et à Paso Coalaque (dépôts post-pliniens).

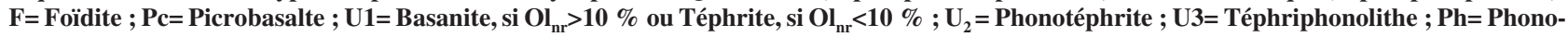
lithe ; $\mathbf{B}=$ Basalte alcalin, si Né ${ }_{\mathrm{nr}}>0$ ou Basalte subalcalin, si Né ${ }_{\mathrm{nr}}<0 ; \mathrm{S} 1=$ Trachybasalte : Hawaïte, $\mathrm{si}\left(\mathrm{Na}_{2} \mathrm{O}-2\right)>\mathrm{K}_{2} \mathrm{O}(\%$-p) ou Trachybasalte potassique, si $\left(\mathrm{Na}_{2} \mathrm{O}-2\right)<\mathrm{K}_{2} \mathrm{O} ; \mathrm{S} 2=$ Trachyandésite basaltique : Mugéarite, si $\left(\mathrm{Na}_{2} \mathrm{O}-2\right)>\mathrm{K}_{2} \mathrm{O}\left(\%\right.$-p) ou Shoshonite, si $\left(\mathrm{Na}_{2} \mathrm{O}-2\right)<\mathrm{K}_{2} \mathrm{O}$; S3= Trachyandésite : Benmoréite, si $\left(\mathrm{Na}_{2} \mathrm{O}-2\right)>\mathrm{K}_{2} \mathrm{O}(\%-\mathrm{p})$ ou Latite, si $\left(\mathrm{Na}_{2} \mathrm{O}-2\right)<\mathrm{K}_{2} \mathrm{O}$; T $=$ Trachyte, si $\mathrm{Q}_{\mathrm{nr}}<20 \%$ ou Trachydacite, si $\mathrm{Q}_{\mathrm{nr}}>20 \%$;

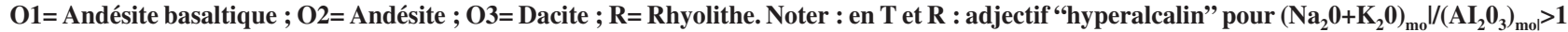
- et en dehors de $\mathrm{S} 1$ à $\mathrm{S3}$ : sodique pour $\left(\mathrm{Na}_{2} \mathrm{O}-4\right)>\mathrm{K}_{2} \mathrm{O}$ et potassique pour $\left(\mathrm{Na}_{2} \mathrm{O}-4\right)<\mathrm{K}_{2} \mathrm{O}$.

Fig. 8: TAS diagram (after Le Bas et al., 1986) showing the geochemical fields of the glasses found in the Sallalli trench and in the Mucurca core, as well as the glasses of the stratotypes of the Huaynaputina deposits at Quinistacas (plinian-fall deposits) and at Paso Coalaque (post-plinian pyroclastic depo-

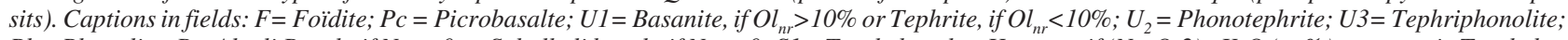
$\mathrm{Ph}=$ Phonolite; $\mathrm{B}=$ Alcali Basalt, if $\mathrm{Ne} \mathrm{e}_{\mathrm{r}}>0$ or Subalkali basalt, if $\mathrm{Ne} \mathrm{e}_{\mathrm{w}}<0 ; \mathrm{SI}=$ Trachybasalte: Hawaïte, if $\left(\mathrm{Na}_{2} \mathrm{O}-2\right)>\mathrm{K}_{2} \mathrm{O}(\mathrm{w}-\%)$ or potassic Trachybasalt, if $\left(\mathrm{Na}_{2} \mathrm{O}-2\right)<\mathrm{K}_{2} \mathrm{O} ; \mathrm{S} 2=$ Basaltic trachyandesite: Mugearite, if $\left(\mathrm{Na}_{2} \mathrm{O}-2\right)>\mathrm{K}_{2} \mathrm{O}(w-\%$ - $p)$ or Shoshonite, if $\left(\mathrm{Na}_{2} \mathrm{O}-2\right)<\mathrm{K}_{2} \mathrm{O}$; $\mathrm{S} 3=$ Trachyandesite: Benmoreit, if $\left(\mathrm{Na}_{2} \mathrm{O}-2\right)>\mathrm{K}_{2} \mathrm{O}(w-\%)$ or Latite, if $\left(\mathrm{Na}_{2} \mathrm{O}-2\right)<\mathrm{K}_{2} \mathrm{O} ; \mathrm{T}=$ Trachyte, if $Q_{n r}<20 \%$ or Trachydacite, if $Q_{n r}>20 \%$; O1= Andésite basaltique; O2=

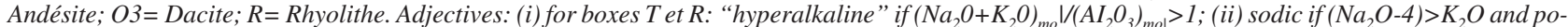
tassic if $\left(\mathrm{Na}_{2} \mathrm{O}-4\right)<\mathrm{K}_{2} \mathrm{O}$ (not available for boxes $\mathrm{S} 1, \mathrm{~S} 2$ and $\mathrm{S} 3$ ). 
hétérogène, qui comprend l'ensemble des produits existant dans les strato-volcans locaux ainsi que dans les dépôts dispersés aux alentours par le vent et le ruissellement. Dans ces conditions la distinction entre téphra et dépôts volcanoclastiques est avant tout fondée sur l'isolement dans les séquences tourbeuses et la netteté des contacts entre les deux matériaux.

\section{1 - LES TÉPHRAS À VERRES RHYOLITHIQUES}

Des niveaux de microponces et verres ont été trouvés dans trois sites : la tranchée ( $\mathrm{Tr}-\mathrm{T} 1$ ) et le sondage $\mathrm{S} 2$ de Sallalli (S2-T1), ainsi que la Laguna Mucurca (LMT1). Tous ces verres ont la même composition chimique de rhyolithe à tendance potassique (tab. 2, fig. 8). Malgré l'identité géochimique des verres de la tranchée et du sondage S2 de Sallalli, leurs âges ${ }^{14} \mathrm{C}$ respectifs excluent qu'ils soient mis en corrélation.

Dans la tranchée de Sallalli, la datation ${ }^{14} \mathrm{C}$ obtenue pour Tr-T1 délimite la période comprise entre 1460 et 1670 cal AD pendant laquelle une seule retombée ponceuse très largement dispersée est connue, celle du Huaynaputina survenue en février-mars 1600 AD (Navarro Oviedo et al., 2000).

Dans la carotte S2, le téphra S2-T1 est encadré par deux âges ${ }^{14} \mathrm{C}$ qui le situent entre $130 \mathrm{cal} \mathrm{AD}$ et $800 \mathrm{cal}$ BC. Pendant cette période une éruption plinienne du Misti a eu lieu ; elle a été datée par Thouret et al. (1995) à $1920 \pm 200 \mathrm{aBP}$, puis redatée plus précisément à $2050 \mathrm{aBP}$ à Arequipa par Thouret et al. (2001), soit entre 400 cal BC et 340 cal AD (nous écrirons Misti$\mathrm{BC} / \mathrm{AD}$ pour indiquer que cette éruption se situe aux environs du passage à l'ère chrétienne).

Dans la carotte de Mucurca, le seul âge ${ }^{14} \mathrm{C}$ disponible est de 920-760 cal BC à 210-225 cm, alors que les verres LM-T1 se trouvent à $120 \mathrm{~cm}$ de profondeur ; ici la corrélation est donc possible tant avec l'éruption du Huaynaputina-1600 AD qu'avec celle du Misti$\mathrm{BC} / \mathrm{AD}$, mais en cas de vitesse de sédimentation constante au cours des deux derniers millénaires, l'appartenance au Misti prend l'avantage.

Si l'on compare la composition des verres à celle des produits du Huaynaputina-1600AD dans les deux stratotypes proches du volcan (Loutsch, 1999), alors, ils ne peuvent correspondre qu' aux produits de la phase postplinienne (tab. 2, fig. 8). Aucune comparaison n'est possible avec les produits pliniens du Misti-BC/AD, car les analyses des ponces de ce dernier ont été pratiquées uniquement en roche totale (tab. 2). Par ailleurs, des analyses 'roche totale' des produits du Huaynaputina-1600AD ont montré que le magma est dacitique (Loutsch, 1999 ; Thouret et al., 1999, 2002a ; Adams et al., 2001), donc normalement moins différencié que le verre. Quand il s'agit des produits du Misti-BC/AD, il faudrait donc raisonner avec des écarts non rigoureux pour discuter l'origine des verres.

Par ailleurs, le minéral mafique dominant dans les stratotypes des produits du Huaynaputina-1600AD est l'amphibole (environ $90 \%$ ), suivie de l'apatite, puis seulement les deux pyroxènes (Loutsch, 1999). A Sallalli, dans Tr-T1, il n'y a que $21 \%$ d'amphiboles, les pyroxènes sont dominants, et il n'y a pas d'apatite. Il faut aussi signaler que le tri sélectif, lors du transport atmosphérique du Huayanaputina à Sallalli (environ $120 \mathrm{~km}$ ), aurait dû augmenter la part d'amphibole par rapport aux pyroxènes (Juvigné, 1983). Enfin, il faut encore remarquer que l'association de minéraux denses de Tr-T1 est identique à celle de S2-T1. Si le téphra Tr-T1 provient du Huaynaputina-1600AD, sa composition minéralogique ne peut s'expliquer par sa contamination par des minéraux locaux lors du remaniement éolien dont la retombée primaire a été l'objet peu après sa retombée (voir plus haut).

En conclusion, sur la base de l'âge ${ }^{14} \mathrm{C}$ et de la composition des verres, le téphra Tr-T1 de la tranchée de Sallalli devrait correspondre aux produits post-pliniens de l'éruption du Huaynaputina-1600AD, mais il faut alors admettre une contamination in situ pour expliquer la pauvreté en amphiboles de l'association de minéraux denses. Par ailleurs, le téphra S2-T1 de Sallalli devrait provenir d'une éruption du Misti-BC/AD. Quant au niveau de verres rhyolithiques de Mucurca (LM-T1), il s'accommode davantage de l'éruption du Misti$\mathrm{BC} / \mathrm{AD}$. En défendant l'hypothèse de deux retombées ponceuses dans la région, il faut alors s'étonner des lacunes stratigraphiques qui font que les deux téphras n'ont été trouvés ensemble dans aucun des sites étudiés. Rappelons ici que ce sont deux âges ${ }^{14} \mathrm{C}$ incompatibles qui empêchent la corrélation globale des trois occurrences de verres rhyolithiques.

\section{2 - TROIS NOUVEAUX TÉPHRAS D'ORIGINE PHRÉATOMAGMATIQUE}

La carotte du sondage S3 de la tourbière de Sallalli contient trois téphras bien individualisés dans la tourbe. Il constitue ainsi la séquence téphrostratigraphique de référence. Par comparaison avec le téphra récent du Sabancaya, leur composition minéralogique variée atteste leur origine phréatomagmatique, et leur granularité cendreuse est en faveur d'une origine locale (Ampato ou Sabancaya). Leurs âges respectifs sont : S3-T1, entre environ 150 cal AD et 100 cal BC ; S3-T2, entre environ 150 cal $\mathrm{BC}$ et $420 \mathrm{cal} \mathrm{BC}$; S3-T3, entre environ $2100 \mathrm{cal} \mathrm{BC}$ et $2500 \mathrm{cal} \mathrm{BC}$.

\section{3 - LE SECTEUR DU RIO SALLALLI}

Téphrostratigraphie. Dans la carotte S2, le sable gravillonneux S2-S1 qui recouvre le téphra Misti-BC/AD ne peut guère être attribué qu'au ruissellement, car le site est en bordure de la tourbière ; ce matériau est nécessairement postérieur à l'éruption du Misti$\mathrm{BC} / \mathrm{AC}$, il est particulièrement grossier et n'a été trouvé nulle part ailleurs.

Quant aux trois niveaux de sable qui se trouvent dans la séquence de gyttja du sondage $\mathrm{S} 2$ et sous la tourbe 
datée entre 800 cal BC et 200 cal AD, ils peuvent être corrélés comme suit :

- S2-S2 (205-210 cm) avec S3-T1 (entre environ $150 \mathrm{cal} \mathrm{AD}$ et $100 \mathrm{cal} \mathrm{BC})$;

- S2-S3 (245-255 cm) avec S3-T2 (entre environ $150 \mathrm{cal} \mathrm{BC}$ et $420 \mathrm{cal} \mathrm{BC)}$;

- S2-S4 (266-280 cm) avec S3-T3 (entre environ $2100 \mathrm{cal} \mathrm{BC}$ et $2500 \mathrm{cal} \mathrm{BC}$ ).

Cette tentative de corrélation entre les sites S2 et S3 implique que les téphras correspondants soient également présents dans la séquence de la tranchée et de la carotte S1 qui remontent jusqu'à environ $8100 \mathrm{cal} \mathrm{BC}$. Or, une seule lentille sableuse de composition minéralogique adéquate y a été reconnue sous le téphra du Huaynaputina (coupe C). Il faut donc accepter des lacunes stratigraphiques dans la tranchée et le sondage S1.

Géomorphologie dynamique. La coupe transversale recoupant les observations faites sur les deux versants du vallon du Rio Sallalli (fig. 4) montre que leurs couvertures tourbeuses respectives correspondent à des périodes très différentes: entre environ 9000 et $7000 \mathrm{cal} \mathrm{BC}$ en rive gauche, et la période d'environ $8000 \mathrm{cal} \mathrm{BC}$ à aujourd'hui sur un replat de rive droite. Cette situation interpelle le géomorphologue.

L'actuel fond de vallée du Rio Sallalli à l'endroit de la coupe de rive gauche était le fond d'une dépression thermokarstique (kettle hole) lors de la déglaciation, la formation de la tourbe y a été intense au début de l'Holocène et la retombée d'un téphra d'origine phréatomagmatique y a eu lieu (Juvigné et al., 1998). L'épaisseur de tourbe a dû y être supérieure à l'actuelle.
Dans cette zone à peine libre de glace, le Rio Sallalli se mettait en place sous la forme de déversements lacustres en série d'un kettle hole à l'autre. Il a alors dérivé sur le versant droit de sa vallée, en glissant vers sa gauche (vers l'Est) et en érodant la tourbe supérieure du kettle hole. C'est aux environs de 7000 cal BC que le ruisseau s'est encaissé à l'endroit de son lit actuel. Dans ce modèle d'évolution, la tourbification a pratiquement cessé en rive gauche en raison du drainage favorisé par une forte dénivelée, tandis qu'en rive droite, elle se poursuivait sur une pente plus faible, voire un replat.

\section{4 - UN TÉPHRA PHRÉATOMAGMATIQUE DANS LA LAGUNA MUCURCA}

Le niveau du lac a nécessairement été plus élevé qu'aujourd'hui après la période 920 à $760 \mathrm{cal} \mathrm{BC}$ (cf. âge ${ }^{14} \mathrm{C}$ ) pour permettre une sédimentation lacustre sur plus de $225 \mathrm{~cm}$ à l'endroit du sondage. Ce haut niveau a dû exister jusqu'à un moment très récent, puisque après la retombée du téphra à verres rhyolithiques (Misti-BC/AD ou Huaynaputina-1600AD), $120 \mathrm{~cm}$ de dépôts lacustres se sont encore accumulés. Le bas niveau actuel du lac serait donc extrêmement récent, voir partiellement saisonnier.

Dans la logique de la ressemblance des éléments de description du sable gravillonneux de Mucurca LM-S1 avec ceux du téphra récent du Sabancaya, ce matériau peut aussi être considéré comme un téphra d'une éruption phréatomagmatique. D'après l'âge du sédiment

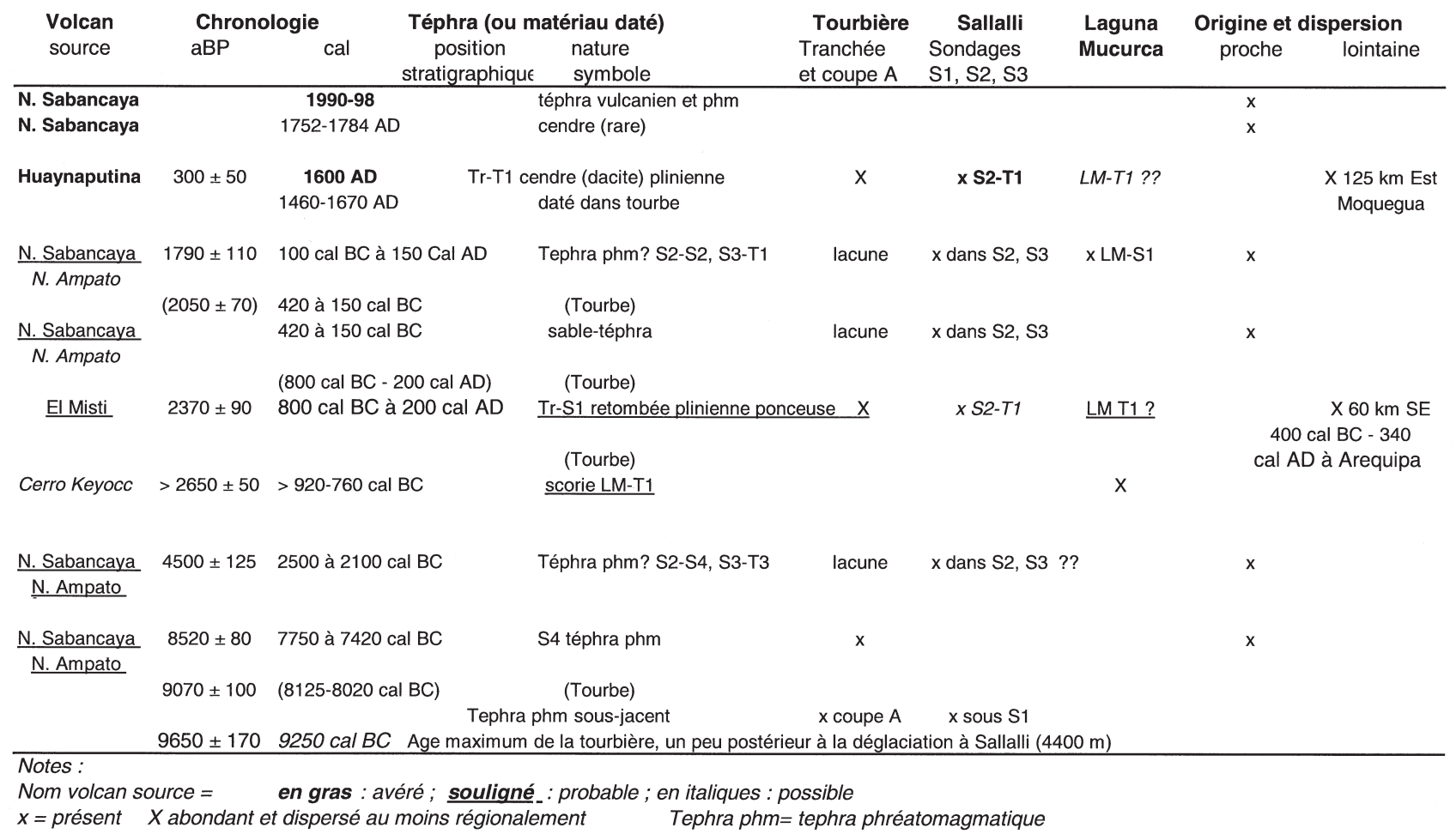

Tab. 4 : Volcans sources et téphras datés : corrélations locales et régionales proposées.

Tab. 4: Volcanoes identified as sources and dated tephras: proposed correlations at local and regional scales. 
lacustre immédiatement sus-jacent, elle aurait eu lieu au début du dernier millénaire BC ou peu avant. Pour établir une corrélation avec le site de référence $\mathrm{S} 3$ et le téphra S3-T3 de la tourbière de Sallalli, il faudrait remonter à environ 2100-2500 cal BC, mais le matériau est plus grossier ici. Cependant, il existe des cônes stromboliens à seulement $4 \mathrm{~km}$ au nord de la lagune, et parmi eux, le cône Keyocc qui est le plus proche (fig. 2) et le plus récent. C'est cette seconde hypothèse que nous privilégions ici en raison de la forte épaisseur et de la granularité de lapilli du matériau.

\section{7 - CONCLUSIONS}

Dans la région du massif des Nevados Ampato Sabancaya et Hualca Hualca (Cordillère occidentale au sud du Pérou), une tranchée et quatre sondages ont permis d'investiguer la période comprise entre $9250 \mathrm{cal} \mathrm{BC}$ et aujourd'hui, et plusieurs téphras ont été mis en évidence (tab. 4).

Un niveau de verres rhyolithiques qui se présente dans trois sites ne peut être attribué avec certitude à l'une ou l'autre des deux éruptions pliniennes récentes connues (Huaynaputina-1600 AD ou Misti-BC/AD).

Tous les téphras plus anciens ont la propriété commune d'avoir une composition très hétérogène manquant d'originalité minéralogique, puisqu'ils contiennent l'ensemble des produits existant dans les strato-volcans Ampato et Sabancaya, et de ce fait dans tous les dépôts périphériques. Ils devraient correspondre à des faciès de débouchage et/ou des éruptions phréatomagmatiques. Le plus ancien a été émis lors d'une éruption du Nevado Sabancaya vers 8500 cal BC. Les suivants datent successivement des périodes suivantes : 2100 à 2500 cal $\mathrm{BC}, 150$ à 420 cal $\mathrm{BC}$ et $150 \mathrm{cal} \mathrm{AD}$ à 100 cal BC.

Dans la Laguna Mucurca, un téphra d'origine phréatomagmatique a également été trouvé, qui est de peu antérieur à la période comprise entre 920 à 760 cal BC, et il a probablement été émis par le cône Keyocc, tout proche.

Les lacunes stratigraphiques avérées dans les différents sites étudiés portent à croire que la récurrence de l'activité volcanique holocène dans le Massif de l'Ampato-Sabancaya est plus fréquente (mais de volume limité) que ne le montrent les résultats acquis dans la présente étude.

\section{REMERCIEMENTS}

Nous remercions les partenaires péruviens de l'Instituto Geofísico del Perú et de Ingemmet, ainsi que l'Institut de Recherche pour le Développment (IRD) au Pérou, qui ont appuyé le travail de terrain, ainsi que la Commission on Tephrochronology and Volcanism (COTAV-INQUA), qui a soutenu le programme TESSOPE au Sud du Pérou de 1995 à 1999.

\section{RÉFÉRENCES BIBLIOGRAPHIQUES}

ADAMS N.K., DE SILVA S., SELF S., SALAS G., SCHUBRING S., PERMENTER J.-L., \& ARBESMAN K., 2001 - The physical volcanology of the 1600 eruption of Huaynaputina, Southern Peru. Bulletin of Volcanology, 62, 493-518.

COBEÑAS G., 2007 - Etude pétrologique des produits éruptifs holocènes du volcan El Misti. Travail d'Etude et de Recherche de Master 1 Géologie (non publié). LMV, Université Blaise Pascal, 25 p.

DA SILVA I., \& ZIELINSKI G., 1998 - Global influence of the AD 1600 eruption of Huyanaputina, Peru. Nature, 393, 397-498.

DELACOUR A., GERBE M.-C., THOURET J.-C., WORNER G., \& PAQUEREAU P., 2007 - Magma evolution of Quaternary minor volcanic centres in Southern Peru, Central Andes. Bulletin of Volcanology, 69, 6, 581-606.

GERBE M.-C., \& THOURET J.-C., 2004 - Role of magma mixing in the petrogenesis of lavas erupted through the 1990-98 explosive activity of Nevado Sabancaya in South Peru. Bulletin of Volcanology, 66, 541-561.

JUVIGNÉ E., 1983 - Les variations minéralogiques dans les retombées de 1982 du volcan El Chichon (Chiapas, Mexique) et leur intérêt pour la téphrostratigraphie. Annales de la Société Géoogique de Belgique, 106, 311-325.

JUVIGNÉ E., THOURET J.-C., GILOT E., GOURGAUD A., LEGROS F., URIBE M., \& GRAF K., 1997 - Etude téphrostratigraphique et bioclimatique du Tardiglaciaire et de l'Holocène de la Laguna Salinas, Pérou méridional. Géographie Physique et Quaternaire, 51 (2), 219-231.

JUVIGNÉ E., THOURET J.-C., GILOT E., LECLERCQ L., \& GOURGAUD A., 1998 - L'activité du volcan Nevado Sabancaya (Pérou) au cours de l'Holocène. « Hommes et Volcans », Quaternaire, 9 (1), 45-51

LAMADON S., 1999 - Fluctuations glaciaires et téphrostratigraphie dans les montagnes intertropicales : une revue et application dans les Andes du Sud Pérou (massifs des Nevados Ampato et Coropuna). Mémoire de DEA (non publié), Université Blaise Pascal, 180 p.

LEAKE B.E., BIRCH W.D., GILBERT M.C., GRICE J.D., HAWTHORNE F.C., KATO A., KISH H.J., KRIVOVICHEV V.G., LINTHOUT K., LAIRD J., MARESH W.V., NICKEL E.H., ROCK N.M.S., SCHUMACHER J.C., SMITH, D.C., STEPHENSON N.C.N., UNGARETTI L., WHITTAKER E.J.W., \& YOUZHI G., 1997 - Nomenclature of amphiboles. Report of the Subcommittee on Amphiboles of the International Mineralogical Association Commission New Minerals and Mineral Names. European Journal of Mineralogy, 9, 623-651.

LE BAS M.J., LE MAITRE R.W., STRECKEISEN A., ZANETTIN B., \& IUGS Subcommission on the Ssytematics of Igneous Rocks, 1986 - A chemical classification of volcanic rocks based on the total Alkali-Silica Diagram. Journal of Petrology, 27, 745-750.

LOUTSCH I., 1999 - Récurrence de l'activité volcanique au Pérou méridional durant les trois derniers millénaires. Mémoire Licence de géographie, Laboratoire de géomorphologie, Université de Liège, 71 p.

MARK B.G., SELTZER G.O., \& RODBELL D.T., 2004 - Late Quaternary Glaciations of Ecuador, Peru and Bolvia. In J. Ehlers \& P.L. Gibbard (eds.), Quaternary glaciations - Extent and chronology, Parti III: South America, Asia, Africa, Australasia, Antarctica. Developments in Quaternary Science, Elsevier, Amsterdam, Vol. 2, 151-163.

MORIMOTO N., 1989 - Nomenclature of Pyroxenes. Canadian Mineralogist, 27, 143-156.

NAVARRO OVIEDO R., JARA R.L.A., THOURET J.-C., SIEBE C., \& DÁVILA J., 2000 - The AD 1600 eruption of Huanynaputina as described in early Spanish chronicles (translated from: "La reventazón del Huaynaputina en 1600", In "Antología del valle de Omate” por R. Navarro, 1994). Boletìn Sociedad geológica del Perú, 90, 121-132.

RISACHER F., 1992 - Les salars de l'altiplano de Bolivie. La Vie des Sciences, Gauthier-Villars, Paris, 9, 1, 39-62.

RISACHER F., \& FRITZ B., 1991 - Quaternary geochemical evolution of the salars of Uyuni and Coipasa, Central Altiplano, Bolivia. Chemical Geology, 90, 211-231. 
SMITH J.A., SELTZER G.O., FARBER D.L., RODBELL D.T., \& FINKEL R.C., 2005 - Early local Last Glacial Maximum in the tropical Andes. Science, 308, 5722, 678-681.

STUIVERT M., REIMER P.J., \& BRAZIUNAS T.F., 1998 - Highprecision radiocarbon age calibration for terrestrial and marine samples. Radiocarbon, 40, 3, 1127-1151.

THOURET J.-C., GUILLANDE R., HUAMAN D., GOURGAUD A., SALAS G., \& CHOROWICZ J., 1994 - L'activité actuelle du Nevado Sabancaya (Sud Pérou) : reconnaissance géologique et satellitaire, évaluation et cartographie des menaces volcaniques. Bulletin de la Société Géologique de France, 1, 165, 49-63.

THOURET J.-C., LEGROS F., GOURGAUD A., SALAS G. JUVIGNÉ E., GILOT E., URIBE M., \& RODRIGUEZ A., 1995 - Un exemple de prévision des risques volcaniques au Pérou méridional (région d'Arequipa), fondé sur l'étude de l'activité éruptive récente du strato-volcan El Misti. Compte-Rendus de l'Académie des Sciences (Géomatériaux), Paris, 320, série IIa, 923-929.

THOURET J.-C., DAVILA J., RIVERA M., EISSEN J.-PH., GOURGAUD A., LE PENNEC J.-L., \& JUVIGNÉ E., 1997 L'éruption explosive de 1600 au Huaynaputina (Pérou), la plus volumineuse de l'histoire dans les Andes centrales. Comptes-Rendus de l'Académie des Sciences (Géomatériaux), Paris, 325, 931-938.

THOURET J.-C., DAVILA J., \& EISSEN J.-PH., 1999 - Largest historic explosive eruption in the Andes at Huaynaputina volcano, south Peru. Geology, 27 (5), 435-438.
THOURET J.-C., JUVIGNÉ E., LOUTSCH I., \& CHAVEZ CHAVEZ J.A., 2000 - Activité volcanique historique et sacrifices humains chez les Incas au Pérou méridional. In E. Juvigné \& J.-P. Raynal (eds.), Tephras, chronologie, archéologie. Les dossiers de l'Archéo-Logis 1. CDERAD, Goudet, 219-226.

THOURET J.-C., SUNI J., FINIZOLA A., FORNARI M., LEGELEY-PADOVANI A., \& FRECHEN M., 2001 - Geology of El Misti volcano near the city of Arequipa, Peru. Geological Society of America Bulletin, 113, 12, 1593-1610.

THOURET J.-C., JUVIGNÉ E., MARIÑO J., MOSCOL M., LOUTSCH I., DÁVILA J., LEGELEY-PADOVANI A., LAMADON S., \& RIVERA M., 2002a - Late Pleistocene and Holocene tephro-stratigraphy and chronology in Southern Peru. Boletín de la Sociedad Geológica del Perú, 93, 45-61.

THOURET J.-C., DÁVILA J., JUVIGNÉ E., GOURGAUD A., \& BOIVIN P., 2002b - Reconstruction of the AD 1600 explosive eruption at Huaynaputina volcano, Peru, based on geologic evidence and Spanish chronicles. Journal of Volcanology and Geothermal Research, 115, 3-4, 529-570.

THOURET J.-C., RIVERA M., WÖRNER G., GERBE M.-C., FINIZOLA A., FORNARI M., \& GONZALES K., 2005 - Ubinas: evolution of the historically most active volcano in Southern Peru. Bulletin of Volcanology, 67, 557-589.

TRAVADA y CÓRDOVA (1752), 1958 - El suelo de Arequipa convertido en cielo (Historia general de Arequipa). Primer festival del libro Arequipeño, edición 1958, 15 p. 